\title{
Analysis and Exploitation of Landforms for Improved Optimisation of Camera- Based Wildfire Detection Systems
}

Andries M. Heyns (D), Department of Science and Technology-National Research Foundation (DST-NRF) Centre of Excellence in Mathematical and Statistical Sciences (CoE-MaSS), Private Bag 3, Wits, Johannesburg 2050, South Africa, Humanitarian Logistics and Supply Chain Research Institute, Supply Chain Management and Social Responsibility, Hanken School of Economics, PO Box 479, 00101 Helsinki, Finland, Laboratory for Location Science, Department of Geography, University of Alabama, Tuscaloosa, AL 35487, USA and University of Pretoria, Lynnwood Road, Pretoria 0002, South Africa

Warren du Plessis (D), University of Pretoria, Lynnwood Road, Pretoria 0002, South Africa

Kevin M. Curtin (D), Laboratory for Location Science, Department of Geography, University of Alabama, Tuscaloosa, AL 35487, USA Michael Kosch (D), South African National Space Agency, Hospital Street, Hermanus 7200, South Africa, Department of Physics, Lancaster University, Lancaster LA1 4YW, UK, University of Western Cape, Robert Sobukwe Road, Bellville, Cape Town 7535, South Africa and EnviroVision Solutions, PO Box 1535, Westville, Durban 3630, South Africa Gavin Hough, EnviroVision Solutions, PO Box 1535, Westville, Durban 3630, South Africa

Received: 4 September 2020/Accepted: 9 March 2021/Published online: 10 April 2021

Abstract. Tower-mounted camera-based wildfire detection systems provide an effective means of early forest fire detection. Historically, tower sites have been identified by foresters and locals with intimate knowledge of the terrain and without the aid of computational optimisation tools. When moving into vast new territories and without the aid of local knowledge, this process becomes cumbersome and daunting. In such instances, the optimisation of final site layouts may be streamlined if a suitable strategy is employed to limit the candidate sites to landforms which offer superior system visibility. A framework for the exploitation of landforms for these purposes is proposed. The landform classifications at 165 existing tower sites from wildfire detection systems in South Africa, Canada and the USA are analysed using the geomorphon technique, and it is noted that towers are located at or near certain landform types. A metaheuristic and integer linear programming approach is then employed to search

\footnotetext{
*Correspondence should be addressed to: Andries M. Heyns, E-mail: andries.heyns@hanken.fi
} 
for optimal tower sites in a large area currently monitored by the ForestWatch wildfire detection system, and these sites are then classified according to landforms. The results support the observations made for the existing towers in terms of noteworthy landforms, and the optimisation process is repeated by limiting the candidate sites to selected landforms. This leads to solutions with improved system coverage, achieved within reduced computation times. The presented framework may be replicated for use in similar applications, such as site-selection for military equipment, cellular transmitters, and weather radar.

Keywords: Fire detection, Maximal cover, Landforms, Facility location, NSGA-II, Integer linear programming

\section{Introduction}

Camera-based wildfire detection system (CWDSs) are comprised of a number of specialised tower-mounted cameras that monitor the surrounding environment with the aim of providing early wildfire detection $[1,2]$. Historically, the candidate and final sites at which to place the towers are identified by foresters and locals with intimate knowledge of the terrain and without significant use of computational tools. Compared to single-site optimisation, where the aim is to find a single optimal site according to individual camera visibility, system-site optimisation is a complex and delicate process because the overall detection potential depends on the combined visibility cover of multiple cameras in the system. When configuring a CWDS, the number of candidate sites at which to place the towers may far outnumber the camera towers available for placement and it is therefore necessary to carefully select a smaller number of final sites from the larger set of candidates. When local knowledge or expertise may not be available - such as when moving into vast and unfamiliar territories - the process becomes even more challenging. A system-site selection framework that alleviates this burden has been developed in collaboration with the South African ForestWatch CWDS, with operations in South Africa, Australia, Spain, Canada and the USA [2]. The principal site requirements of this framework are (a) to minimise the need for user input to select candidate sites, (b) to identify sites that are superior candidates for system-site optimisation, as opposed to single-site optimisation, and (c) to facilitate the monitoring of large territories and therefore the ability to consider a large number of candidate sites.

The process of configuring a CWDS layout is a complex combinatorial optimisation problem, increasing in complexity with an increase in the size of the terrain to monitor and the number of towers to place. Reducing the size of the set of feasible candidate sites - called the placement zone (PZ) - reduces the complexity of the framework's search for the final tower site locations. At the same time, the search efficiency and solution quality may be improved if the PZ is limited to sites that are superior candidates in terms of their potential contribution to overall system detection performance. The process of identifying the candidate sites from which recent CWDS site-selection methods determine final layouts has, however, not received significant attention. This is surprising, given that the quality of the final site layouts can only be as good as the quality of the sites which are considered for selection (garbage in, garbage out). 
The objective in CWDS site selection is visibility-based optimisation and the role that landforms (e.g. peaks, ridges, valleys, slopes) play in the identification of candidate sites is well-documented [3-7]. Ridges and peaks are consistently considered to offer superior observer visibility compared to sites classified otherwise and reducing the PZ to these landforms is therefore expected to result in improved detection capability of the CWDSs obtained by the solution framework. Existing CWDS site-selection methods that do consider landforms are either manual and subjective, without the use of landform classification methods, and applied to unrealistically small hypothetical study areas [8,9], or otherwise meant for singlesite optimisation $[9,10]$. These approaches are therefore not considered suitable for use with the site-selection framework followed in this paper, which is designed for system-site optimisation in significantly larger territories - the study area considered in this paper covers approximately $1505 \mathrm{~km}^{2}$, compared to those smaller than $15 \mathrm{~km}^{2}$ in the literature $[8,9]$.

The purpose of this paper is to examine landform exploitation for system-site optimisation, specifically in large territories, and the main contributions are summarised below.

- Landforms are determined and analysed at 165 actual ForestWatch tower sites from systems in South Africa, Canada and the USA. To the authors' knowledge, such a practical classification exercise of existing visibility-based facilities has not been performed in the literature, and reveals interesting facts about the preferences of decision makers and the compromises they consider when determining tower sites.

- Landform characteristics which are common between the existing sites and sites determined by optimisation (before landform consideration) are identified - the first comparison of its kind in the literature. The results are exploited in order to reduce the size of the PZ to selected landforms.

- It is demonstrated that landform exploitation can be effectively used to enhance location optimisation, particularly when the problem instance is outside the bounds of tractability for global optimisation. The technique and framework developed here may be similarly beneficial to other site-selection problems, e.g. cellular transmitters [11-13], weather radar [14, 15], and military equipment such as signal jammers [16], and radars and weapons [17, 18].

The remainder of the paper is organised as follows. In Sect. 2, background information is provided on terrain modelling, landform analysis, and the processes and considerations related to tower site selection. Section 3 describes the data and methods applied in this paper, including the study areas, visibility analyses, landform classification, and methods of evaluating and optimising CWDS layouts. Section 4 demonstrates the results of the classification of existing and optimisationdetermined tower site classifications, in addition to the results of tower sites obtained after landform exploitation. In Sect. 5, the results are analysed and limitations and potential improvements are discussed. The paper closes with a brief conclusion in Sect. 6. 


\section{Background}

\subsection{Modelling the Physical Environment}

Modelling the earth's surface elevation and related geographical/environmental information can be achieved by employing uniformly spaced, satellite-measured points across the terrain surface - called raster data - and is a standard approach used in the literature for solving facility location problems [7, 19-21]. A strength of the raster data structure is its structural simplicity, ease of implementation, and additionally, the landform classification approach employed in this paper is rasterbased. A raster data representation of a hypothetical terrain is provided in Fig. 1, where the dots on the terrain surface represent the uniformly spaced satellite-sampled elevation data from which the surface is generated. The non-contiguous blue area is an example of terrain identified as suitable for the placement of towers, typically limited to criteria such as allowable geographical and administrative/municipal boundaries and suitable terrain characteristics (e.g. slope) - candidate site selection criteria and processes are described in more detail in Sect. 2.3. The green area is an example area of interest which, in the context of this paper, is typically land belonging to forestry clients. The sites that may be considered for facility placement (the blue dots in the figure) collectively form the PZ.

Shuttle radar topography mission (SRTM) elevation data were used in this paper (obtained from https://dwtkns.com/srtm30m/). SRTM data constitutes satellite-sampled points obtained during an 11-day shuttle mission in February 2000, and was a collaborative effort between agencies from the USA, Germany, and Italy [22]. In general, the distance between neighbouring sample points is approximately $30 \mathrm{~m}$ at the highest resolution, and this resolution was used for all analyses in this paper.

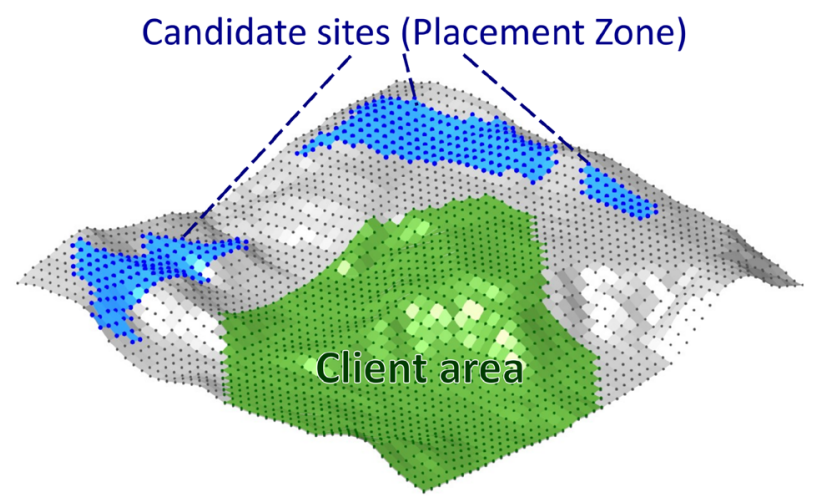

Figure 1. Uniformly spaced sample points of the earth's surface constiłułe raster dała [2] [Image used with copyright permission]. An example of a PZ and client area in raster form is illustrated on the surface. 


\subsection{Landform Classification}

The concept of classifying digitised terrain data (such as the raster surface in Fig. 1) according to spatial patterns goes at least as far back as 1967 [23]. Actual attempts to identify landforms such as pits, peaks, ridges and ravines were performed as early as $1975[24,25]{ }^{1}$ Such morphographical representations of the terrain reveals more detailed characteristics of its appearance and shape than a standard elevation model [26]. A significant portion of landform extraction research is focused towards hydrological applications [26-29] — in fact, many classification approaches make use of hydrological analysis techniques in the process of identifying landforms. Schillaci et al. [26] identify numerous examples of such applications, including drainage pattern extraction and river morphology [25, 3032] and watershed delineation [33, 34]. Other examples identified by Schillaci et al. that are not specifically related to hydrological applications include surface roughness assessment [35], the monitoring of slope movements [36], and predicting the spatial distribution of gully erosion and soil texture [37].

Various classification approaches were recently investigated and compared by Romero and Clarke [38] for open-source and commercial software, and Schillaci et al. [26] provide additional comparisons of classification methods and software. The methods that they investigated require separate workflows in order to identify different landform types, and generally require workflows comprising multiple steps for each. One example of such multiple workflows, and specifically related to our purposes, is the process that Eugenio et al. [10] followed to identify ridges for watchtower placement. Their interesting approach is based on hydrological analyses that are not specifically intended for the purpose of identifying peaks or ridges. Essentially, the digital terrain model is inverted, after which a watershed analysis is performed. A watershed analysis determines where water on terrain will flow and accumulate - when inverted, the original ridges and peaks become river courses and pits, where the water will flow and accumulate.

An alternative landform classification technique is the implementation of predefined terrain patterns that may be matched to continuous land surfaces according to similarities in their geometry $[28,39]$. The ten most significant landform classes - termed geomorphons - were identified by Stepinski and Jasiewicz [39] as flats, peaks, ridges, shoulders, spurs, slopes, pits, valleys, footslopes and hollows, as illustrated in Fig. 2. Their classes were based upon fifteen such pre-defined landform classes first presented and investigated by Schmidt and Hewitt [28], which were based upon the pioneering work of Dikau [40] and Wood [41] in developing geomorphon-based classification processes. The geomorphon classification approach has been successfully used in a variety of recent problems, ranging from the characterisation of submarine bedforms [43], topographic modelling for landscape architecture [44], geographical suitability calculations for agent-based simulation [45], and landslide susceptibility mapping [46].

To identify geomorphons, the entire terrain surface (all raster points) is traversed by a moving search region which matches the geometry of the raster points

\footnotetext{
${ }^{1}$ See the publication of Johnston and Rosenfeld from 1975 [24] for a fascinating map representing landforms by alphanumerical characters.
} 


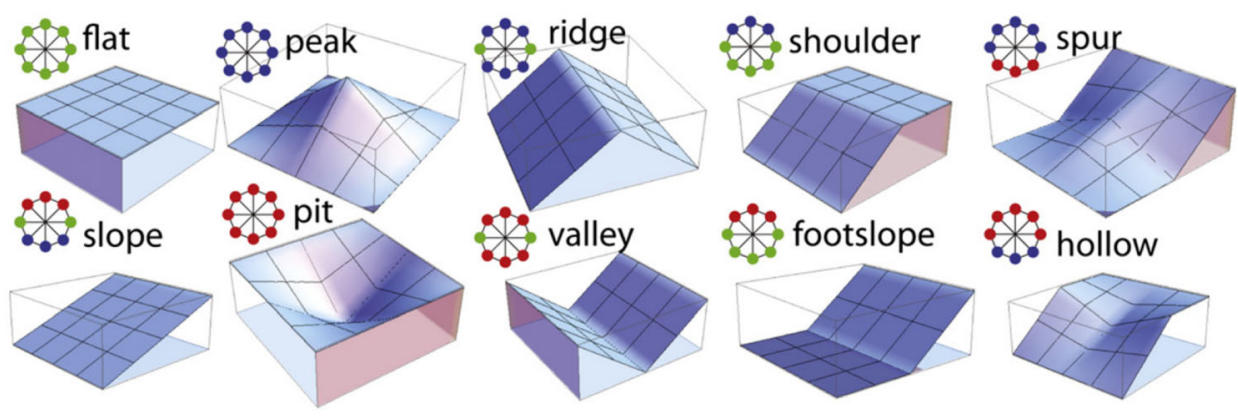

Figure 2. Ten terrain Iandform classifications of [42] [Image used with permission]. The colours of the patterns alongside each class indicate the differences in elevation with respect to the centre point-green indicates same height, red indicates higher, blue indicates lower (Color figure online).

surrounding the centre-point to the pre-defined landform patterns in eight principal directions (N, NE, E, SE, S, SW,W, NW). A ternary operator which employs the three possible elevation differences $(-1$ for lower, 0 for same height, and +1 for higher) is used to identify specific topographic patterns which are associated with the pre-defined landforms in Fig. 2. For example, a ridge is identified when the ternary pattern $[0,-1,-1,-1,0,-1,-1,-1]$ is observed around the centrepoint, when starting from any one of the eight surrounding points and completing a circular visit cycle to the neighbouring points. To provide an illustration of the final result of a geomorphon classification process performed on a digital terrain model, the terrain elevation of the south-eastern part of the study area used in this paper-introduced in more detail later-is displayed in Fig. 3a and its corresponding geomorphon classification is provided in Fig. 3b. The classified areas

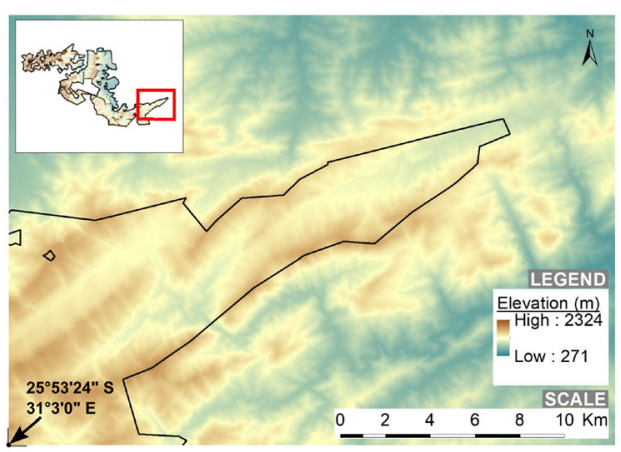

(a)

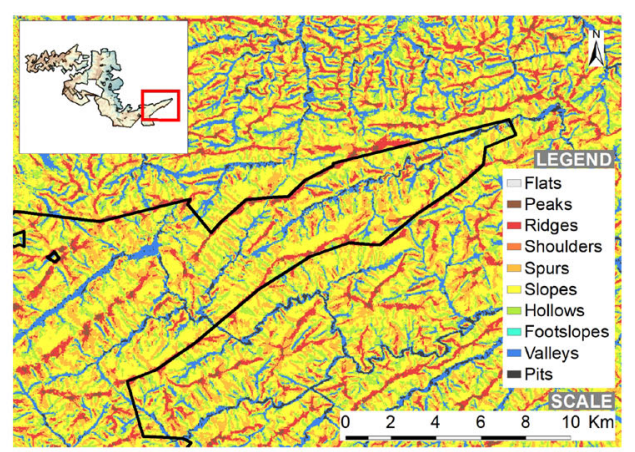

(b)

Figure 3. Example of geomorphon classification results. (a) Terrain elevation of the south-eastern part of the study area used in this paper, and (b) its corresponding geomorphon classifications (determined using 30-m resolution SRTM data). 
are, in fact, the individual raster points' geomorphon classifications (not individually visible due to the scale and the large number of raster points).

The strategy of selecting candidate sites based upon landform classes should be approached carefully. The various classification techniques applied to the same area can return different landform classifications for individual sites, and results returned by a specific technique may also vary with different parameter settings $[38,42,47]$. One example of an important parameter in geomorphon classification is the lookup distance, which is the distance between the centre-point being classified and the surrounding points that are compared to the pre-defined landform patterns - effectively the scale over which landforms are identified [42]. Small lookup distances return landforms identified from a local perspective, while larger values identify landforms from a higher and wider perspective [29]. Suitable lookup distance values vary according to factors such as the resolution of the terrain model, the roughness of the terrain, and the intended purpose of the resulting landform data [42].

Regardless of which technique is employed, its effectiveness for a specific application requires preliminary investigation on a case-by-case basis before preferred landforms can be identified. The purpose of this paper is not to investigate which landform classification technique is "better," but to demonstrate that any landform classification technique can lead to improved optimisation results if the preliminary analyses are adequately performed. Furthermore, the work in this paper is presented in a manner that may be replicated regardless of the landform classification technique employed. The geomorphon process was therefore selected for implementation in this paper-largely due to its simplicity and availability in open-source software. Compared to other landform classification approaches, a major advantage of the geomorphon approach is that a single execution of the process is required to classify all raster points on the terrain surface and therefore requires a single workflow only [42].

\subsection{Candidate Site Selection}

As previously discussed in the introduction, one candidate site selection approach which may be followed is manual selection. In this approach, the terrain's topographical representation (such as the one in Fig. 1) is visually inspected and raster points which are considered to exhibit superior visibility of the area of interest are selected as candidate sites. This approach was used by Bao et al. [8] for the purpose of maximising wildfire detection. They selected 30 candidate tower sites located at what they (subjectively) considered to be peaks and ridges in an area spanning approximately $10 \mathrm{~km}^{2}$ in China. From this set of candidate sites, proposed layouts comprising sites selected from the candidates were determined using combinatorial optimisation approaches (examples of such approaches are provided later). Manual candidate site identification is only practical in such unrealistically small study areas and is not considered desirable for the intended research of this paper, in which expansive terrains with numerous mountains, hills and ridges need to be examined to identify candidate sites. Zhang et al. [9] followed a more computational approach, also for wildfire detection, by first determining the 
visibility of all raster points in their study area of slightly larger than $10 \mathrm{~km}^{2}$, and choosing 34 sites with superior visibility from these as their candidate sites. This process is more computational and user-friendly than a strict manual approach but is, at its core, focussed towards single-site visibility-which does not consider the performance of the sites when combined with others and is not ideal when the ultimate goal is the maximisation of system visibility. This approach is therefore also not considered suitable for system-site optimisation pursued in this paper.

Eugenio et al. [10] followed an approach that employs a geographical information system (GIS) to determine a large number of candidate sites for manned watchtowers in a large area covering $46,000 \mathrm{~km}^{2}$. The PZ was first limited to land within feasible geographical and administrative/municipal boundaries and within suitable distances from roads (for installation and maintenance accessibility), and then reduced to terrain classified as ridges. This method is more suited to implementation with larger areas - and therefore with the framework this paper is based on-since raster points spread across large expanses of terrain can easily and speedily be classified according to practical criteria such as road distance and landform types using a GIS. Their final sites were then selected using an altitudebased elimination approach, discussed in more detail along with other final site-selection approaches in Sect. 2.5. The landform classification approach that they followed (previously detailed in Sect. 2.2) is unconventional and not specifically designed for the identification of ridges, and ridges were selected as preferred landforms without any supporting analysis. Heyns et al. [2] followed a similar candidate site selection process using a GIS. Their PZ was first identified as a number of forestry client areas in their study area in Nelspruit, South Africa, further reduced according to two geospatial criteria. First, terrain with a degree of slope under $12^{\circ}$ (or $20 \%$ ) was selected, which ensures that tower installation may be performed without the need for excessive terrain alteration, in addition to ease of access on foot in the installation area. Second, a distance of $100 \mathrm{~m}$ or less to roads was deemed necessary for transportation (e.g. construction and maintenance) and general access purposes. The resulting PZ - the raster points exhibiting feasible slope and road accessibility within the client areas-is shown in Fig. 4.

A GIS-based candidate site selection approach such as those above is followed in this paper-these approaches typically result in a large number of candidate sites. For example, the approach of Heyns et al. [2] did not employ landform classification to further reduce the size of their PZ and over 700,000 candidate sites are included in Fig. 4. Given the hilly and mountainous nature of this study area, the PZ could be reduced to a considerably smaller number of sites if it is limited to certain landforms only. However, this requires a well-motivated landform classification and exploitation methodology — which remains a strategy to be formally investigated and is the purpose of this paper.

\subsection{Smoke Detection}

While detecting surface flames is one purpose of ForestWatch CWDSs, their focus is on detecting smoke patterns - using a proprietary pattern-recognition algorithm which is based upon South African Antarctic research into the automated detec- 


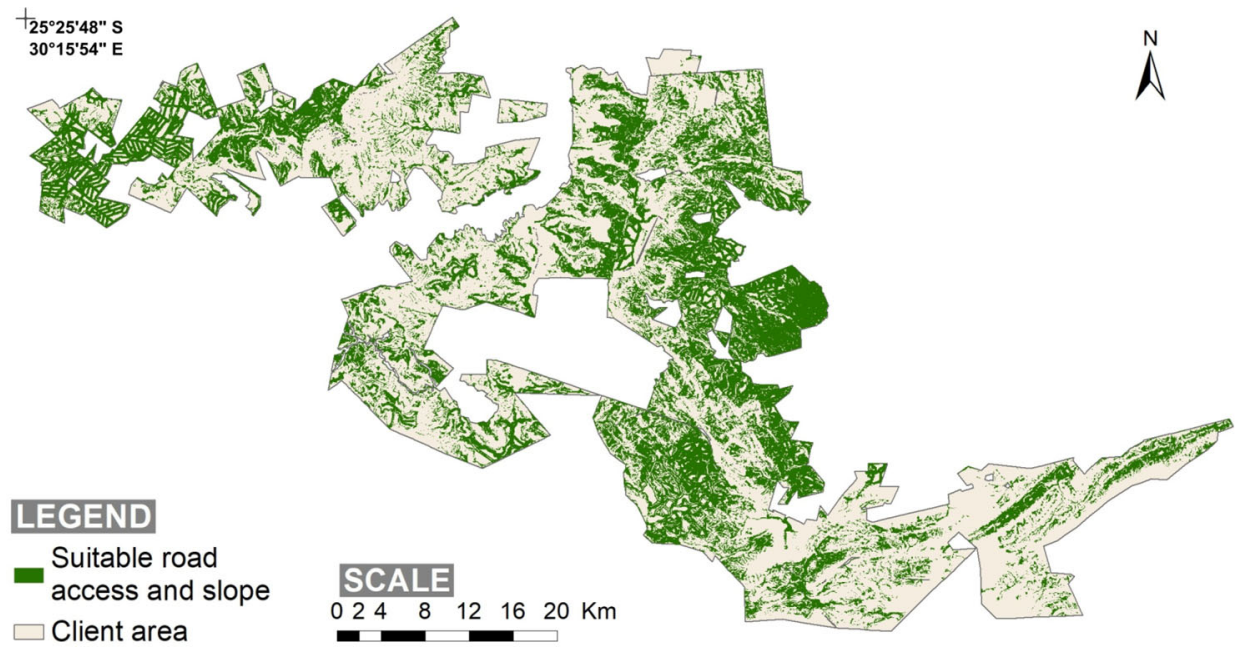

Figure 4. The feasible PZ within the Nelspruit client area in South Africa, where both slope and road access are feasible [2] [Image used with copyright permission]. Landforms were not considered in the determination of this PZ.

tion of aurora [48]. The lower above the terrain surface a smoke plume may be detected, the sooner suppressing action may be taken after the onset of the fire. Terrain and vegetation are, however, more likely to obstruct a camera's visibility of smoke when it is near the terrain surface or when the fire is in a valley or behind a hill. The overall detection potential of a CWDS therefore also depends on its ability to detect smoke at higher levels above the terrain surface (after clearing obstructions). This is a unique approach to fire detection and similar surveillance system applications, where the standard approach is to evaluate visibility with respect to the terrain surface only [7-9, 19, 49]. Furthermore, CWDSs are configured in such a manner that they achieve satisfactory visibility cover over buffer zones added to the smoke layers, for the purpose of detecting fires outside the client area and which may rapidly spread onto client property.

Two smoke detection heights were employed by Heyns et al. [2], each with a different buffer zone size. The same approach is followed in this paper, albeit using different smoke layer heights and buffer zone sizes. A low smoke layer is used for near-immediate detection and rapid client response, and has a smaller buffer zone for detecting fires near the client boundaries which pose an immediate threat of crossing over into client territory. This concept is illustrated in Fig. 5a, where a small buffer is added around a hypothetical client area, after which a low smoke layer with this added buffer is simulated at a low height above the terrain surface-following the contours of the terrain as a layer of smoke would. A higher smoke layer serves the purpose of detecting smoke not detected at the lower layer due to visibility obstructions, and which has risen further to be (potentially) visible, as illustrated in Fig. 5b. Also shown in Fig. 5b is how the higher 


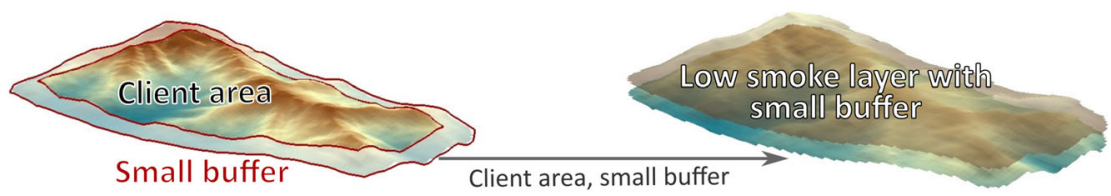

(a)

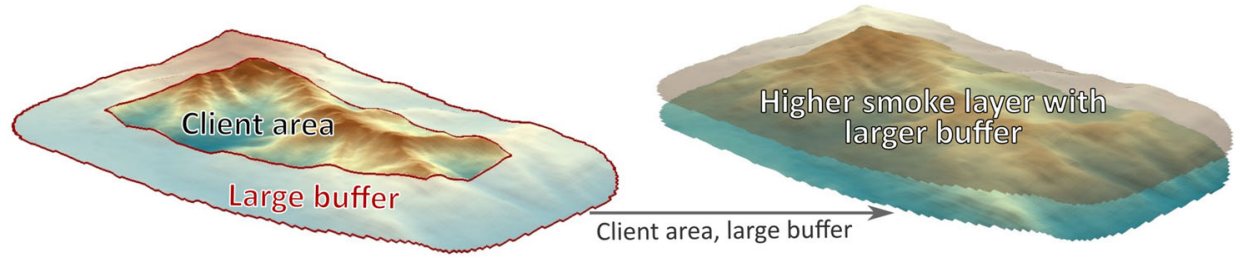

(b)

\begin{abstract}
Figure 5. Buffer zones are added around the client area to monitor threatening external fires, and the combined client and buffer terrains are raised in order to simulate smoke layers at different heights above the terrain surface. (a) A small buffer with a low height is used to determine a CWDS's near-immediate detection capability, and (b) a larger buffer with a higher height is used to evaluate secondary detection potential.
\end{abstract}

smoke layer is associated with an extended buffer zone which allows for the monitoring of fires further outside the client area-these fires need to be monitored, but do not necessarily require immediate response from ForestWatch clients if their property is not under threat. Each of these smoke layers is called a cover zone $(\mathrm{CZ})$, since CWDSs are configured with the aim of maximising their visibility cover with respect to these layers.

\title{
2.5. Optimisation of Final CWDS Site Selection
}

Following the identification of numerous candidate sites in the PZ, determining the precise sites where CWDS towers are to be located is required in order to maximise detection of the CZs. In the related literature (in which only one CZ is considered) one possible approach is incremental site selection, which was investigated by Zhang et al. [9]. This approach places the first tower at the single site which is determined to have the best visibility with respect to the area that requires coverage, the area requiring coverage is updated in light of this tower's coverage, and the process is repeated by adding the tower with the best coverage of the remaining coverage area until all tower positions have been selected. This sequential, greedy single-site optimisation approach is suitable if the intention is to increment system cover by adding towers one at a time, over an extended period of time, but is not suitable for the simultaneous optimisation and placement of numerous towers operating together as a single system. Following their GIS-based 
approach and limiting candidate sites to ridges, Eugenio et al. [10] selected their final sites by dividing their study area into smaller, square sub-regions, and choosing the site with the highest altitude in each sub-region as a tower site. Such an approach is also not recommended because it is intended for single-site optimisation, does not consider a selected site's actual visibility coverage, and is based on the assumption that superior altitude is associated with superior visibility. It has been shown that superior altitude does not necessarily ensure good visibility and a site's relationship to its surrounding environment is crucial $[3,6,7,50]$.

CWDS layout solution approaches which have been used successfully in related research and which are suitable for the problem considered in this paper include integer linear programming (ILP) [8] and heuristics [2, 8] - both approaches are employed in this paper. These methods maximise system visibility with consideration given to the combined coverages of all the towers in the system.

More than one covering objective - one per $\mathrm{CZ}$ - is considered in this paper, and a multi-objective (MO) system-site optimisation approach is therefore followed. Such an approach returns solutions that provide decision-makers with multiple CWDS layouts that exhibit superior trade-offs in the coverages achieved by a system with respect to more than one $\mathrm{CZ}$. An additional and significant benefit to this approach is that each of these proposed layouts offers a unique site configuration-decision-makers may prefer the site locations of some layouts more than those in others, and having multiple layouts allows them to take other issues into consideration as well. In order to achieve such diversity in CWDS coverage and site layouts, a set of solutions that is commonly known in the MO optimisation literature as the Pareto front is desired [51], as displayed by the black markers in Fig. 6. Each of the solutions in the figure represents a candidate CWDS layout,

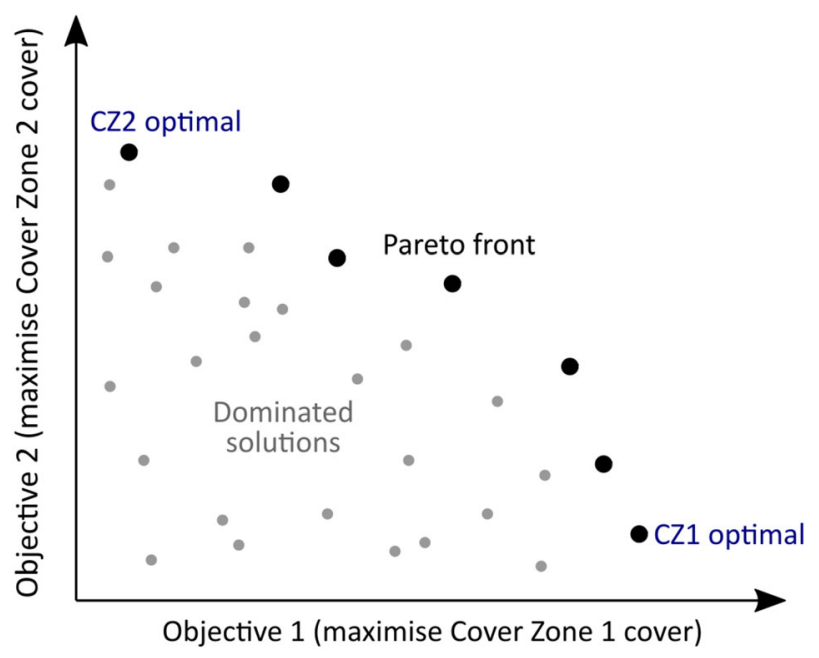

Figure 6. A Pareto front in objective function space, which is sought for decision-making purposes. 
and its location on the graph reflects its coverage achieved with respect to the CZs (typically presented as a percentage of the CZs that are covered). The solutions on the Pareto front outperform (dominate) all the other possible solutions which may exist (such as the grey solutions in the figure) in terms of their trade-offs in cover achieved with respect to the CZs [51].

ILP solvers (such as CPLEX and Gurobi) offer one solution method that may be followed in order to determine solutions on the Pareto front and take as input mathematical formulations of objective functions (here, the maximisation of $\mathrm{CZ}$ detection) and constraints. These solvers are particularly well-suited to determining the end-points on the Pareto front, which are indicated as CZ1 optimal and CZ2 optimal in Fig. 6 - each performing well with respect to one objective, but not with respect to the other. This is because maximising the cover achieved with respect to a single $\mathrm{CZ}$ is less complex than determining those solutions that are found between these two optimal solutions, which require the consideration of more than one $\mathrm{CZ}$ in the optimisation process. Solutions between the single- $\mathrm{CZ}$ ones are obtained using the popular weighted-sum approach [52-54], in which weights are assigned to each $\mathrm{CZ}$ to reflect their relative importance with respect to each other [55]. In the mathematical formulation of the weighted-sum objective, the multiple covering objectives are weighted together into a single objective function, and by varying the $\mathrm{CZ}$ weights in multiple runs the solutions along the front may be "traced out."

ILP solvers are, however, limited by the complexity of the problems which they can solve - in this research the complexity is related to the number of candidate sites and the size of the CZs. Because our framework is aimed at solving large, practical problems, the PZs and CZs considered here are significantly larger than what is generally encountered in facility location problems $[7,8,17,56]$. The use of an ILP is therefore, in general, not possible for the size of the problem in this paper. To overcome this computational limitation, heuristics are often employed in order to approximate the set of solutions on the Pareto front when approaches such as ILP are not possible [51, 57, 58]. The Non-dominated Sorting Genetic Algorithm-II (NSGA-II) is one heuristic that may be employed and has been used extensively in the literature for solving MO optimisation problems, including applications that consider covering objectives $[18,20,56,59]$.

Heyns et al. [2] used the NSGA-II in a two-stage optimisation approach. First, the NSGA-II was used to determine numerous CWDS layouts which outperformed the existing CWDS in their study area in terms of coverage achieved with respect to two CZs. This was an indication that the layouts returned by the heuristic included multiple strong sites. These strong sites were then pooled together into a new PZ, resulting in a smaller sub-set of candidate sites from the original PZ. In the second stage, the NSGA-II was employed once more, using the smaller PZ, and solutions with significant improvements in coverage were discovered. A similar approach is followed here; however, an ILP approach is followed during the second optimisation stage instead. This is because the heuristic stage reduces the number of candidate sites to a number which is small enough for use with an ILP solver. A second and major advantage of the ILP approach, in a practical sense, is that the user is able to specify the desired number of solutions 
(the number of selected weight combinations). Heuristics often generate an impractically large number of solutions on the Pareto front approximation (potentially hundreds or even thousands), which require further analysis to be reduced to a manageable number for decision-makers to analyse and compare [60,61], as was observed in the results of Heyns et al. [2]. The mathematical formulation of the CWDS site-selection problem and the optimisation approach followed in this paper are presented in more detail later.

\section{Data and Methods}

\subsection{Study Areas}

The site locations of 165 actual CWDS towers were obtained from ForestWatch for the classification and analysis of existing sites performed later in this paper. The towers are part of systems in the Mpumalanga Province in South Africa (93 towers), Douglas County in the state of Oregon, USA (31 towers) and the central region of Saskatchewan Province in Canada (41 towers). The layouts of the CWDSs and the surrounding terrain are displayed in Fig. 7.

To evaluate the suitability of landform exploitation for CWDS site selection, a system of twenty six cameras is used as a benchmark to compare and analyse optimisation results, and was previously investigated by Heyns et al. [2]. The system is located in the region of Nelspruit, in the north-east of South Africa, and covers approximately $1505 \mathrm{~km}^{2}$. The system is the highlighted region which may be seen in the centre of Fig. 7a and is shown in more detail in Fig. 8. The relief can be described as rough, with various mountains, hills, valleys, ridges and slopes. The cameras in this system have a specified detection range of $8 \mathrm{~km}$ and are placed on towers that range in height from $12 \mathrm{~m}$ to $54 \mathrm{~m}$ (averaging $42 \mathrm{~m}$ ) at the locations shown in Fig. 8. The cameras have an actual detection range of well beyond $8 \mathrm{~km}$ and fires are often detected at twice this range. An $8 \mathrm{~km}$ range is used for contractual purposes and to mitigate the negative effects of bad weather on practical detection potential.

The Nelspruit PZ (without geomorphon exploitation) which is illustrated in Fig. 4 was determined using ArcGIS 10.5.1 software. Roads shapefiles were provided by ForestWatch to determine distances from roads using the software's Euclidean distance tool, and slope was determined using the software's slope analysis tool. The number of candidate sites in this PZ totalled 741,813. This significant number is a result of the large area and because the terrain model is rasterbased at 30-m intervals.

\subsection{Visibility Analyses}

The detection potential of a CWDS is determined by the coverage it achieves with respect to points within CZs. The smoke layer concept was introduced in Fig. 5, and the CZs are simply the rasterised versions of such smoke layers above the client and buffer areas. This is illustrated in Fig. 9a for a CZ (the brown surface and markers) above the example client area that was introduced in Fig. 1. The portion 


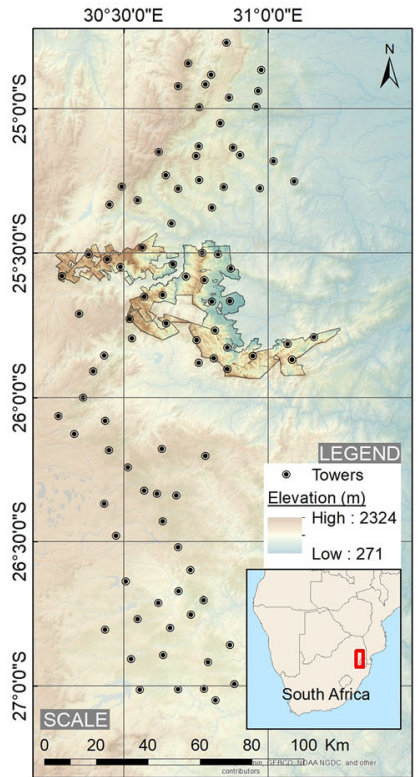

(a)

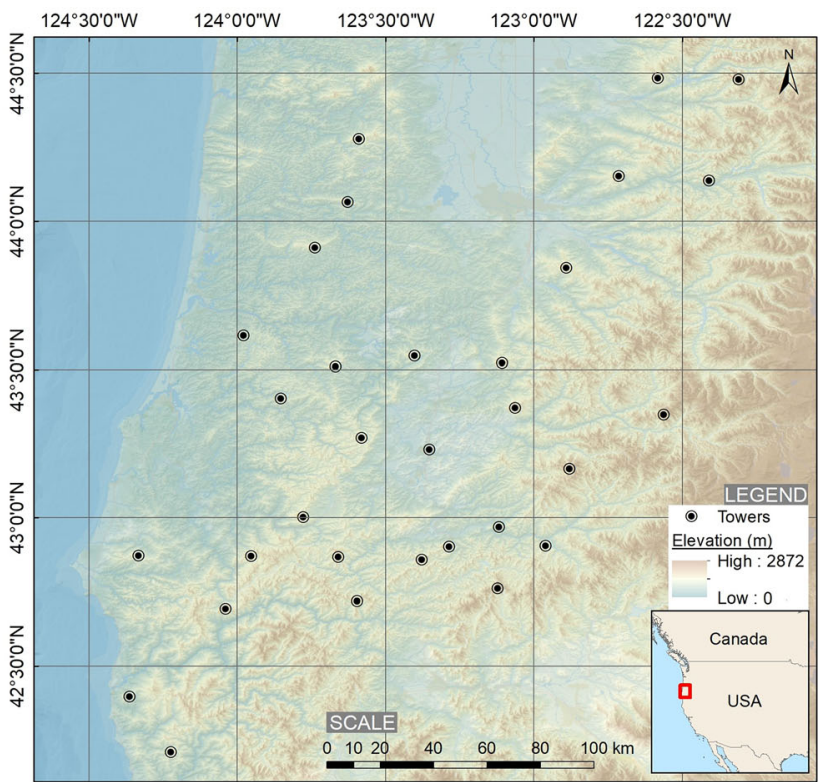

(b)

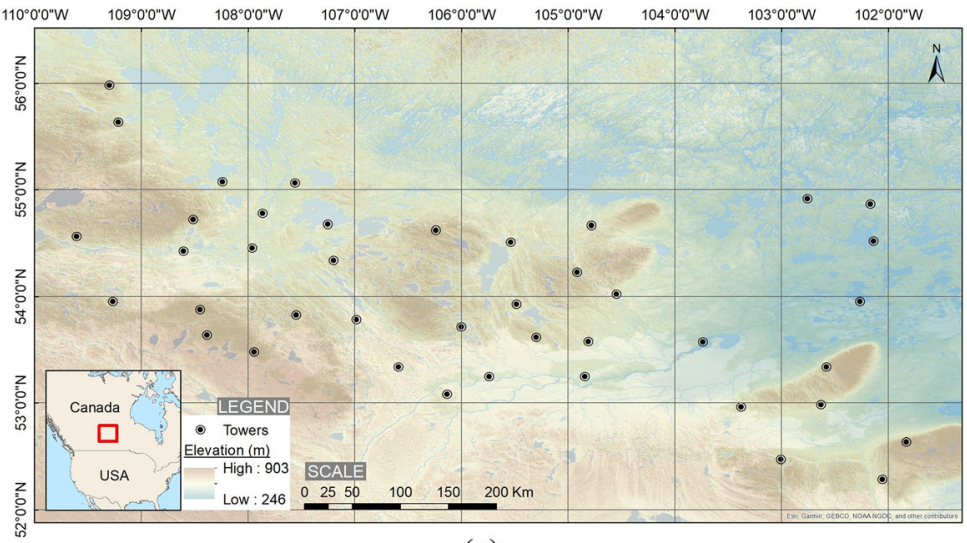

(c)

Figure 7. Landform classifications of towers from three ForestWatch CWDSs are investigated in this paper. (a) Mpumalanga system (93 fowers), (b) Douglas County system (31 towers) and (c) system in the central region of Saskatchewan (41 towers). Sources: Esri, GEBCO, NOAA, National Geographic, DeLorme, HERE, Geonames.org, and other contributors.

of a $\mathrm{CZ}$ that is visible from a camera is referred to as a viewshed [4, 7, 62]. A system viewshed is the merged viewsheds of all the cameras with respect to a specific CZ. A top view of the terrain and CZ in Fig. 9a is provided in Fig. 9b. The com- 


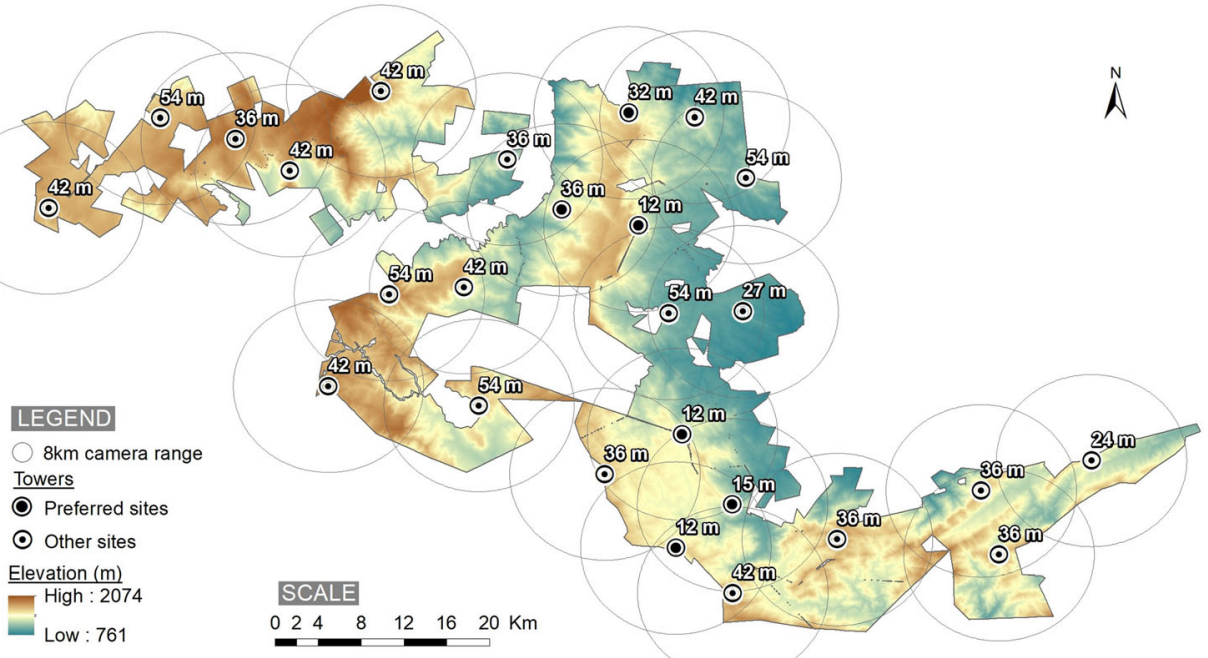

Figure 8. Top view of the ForestWatch CWDS and client area that provided a benchmark for the evaluation of the research in this paper [2] [Image used with copyright permission].

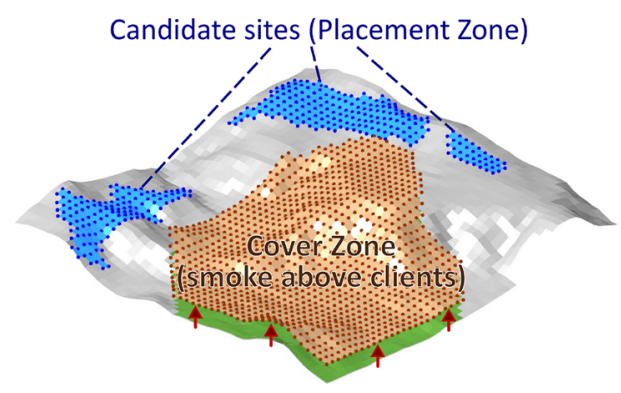

(a)

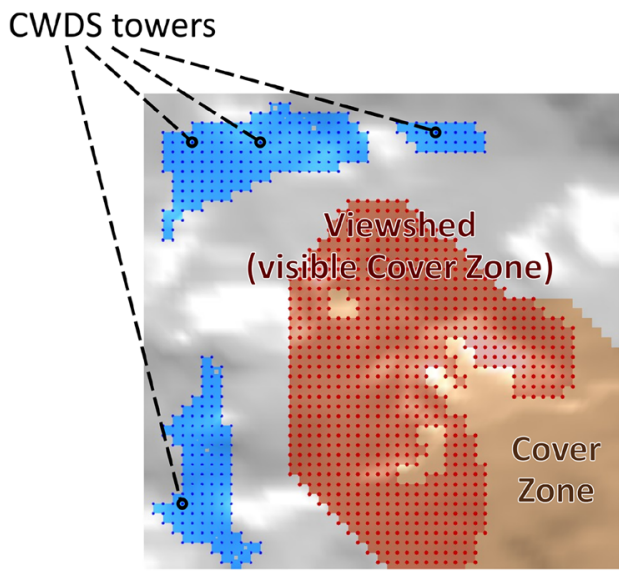

(b)

Figure 9. (a) A CZ above the client area, represented by raster data, (b) top view of the terrain, in which an example of a CWDS tower Iayout (the black markers) and the viewshed it achieves with respect to the CZ (the red area and markers) [2] [Images used with copyright permission] (Color figure online).

bination of the red surface and markers in the figure is an example of a system viewshed achieved by a CWDS comprising four cameras (the black markers). 
A viewshed is determined by a collection of line-of-sight (LOS) queries between the observer and each point in the $\mathrm{CZ}$, returning either 1 if determined to be visible, or 0 otherwise. The Bresenham technique is a classic and simple technique to determine such LOS queries [63, 64], has the advantages of simplicity and efficiency, and is suitable for a raster grid structure. By traversing the raster points along a straight line between the observer and the CZ-point, and comparing the height of the terrain at each raster point with the height of the line between the observer and $\mathrm{CZ}$ point, terrain obstructions may be determined. The authors' own Bresenham line-of-sight code-written and executed within the optimisation processes in the MATLAB software environment discussed later-was used to determine viewsheds in this paper. The coverage achieved with respect to a specific $\mathrm{CZ}$ is expressed as the percentage of the points in the $\mathrm{CZ}$ that are visible.

An important consideration in visibility evaluation is the tower height. Raising a tower's height allows it to see over obstructions such as terrain and vegetation and therefore improves its visibility coverage, and should be incorporated into viewshed computations. The base tower height that was available to planners for the Nelspruit system in Fig. 8 was 12 m, and any increase above this height (typically added in $3 \mathrm{~m}$ increments) depended on a) site suitability to accommodate an increase in tower size and resulting structural support, b) visibility obstruction caused by surrounding vegetation (e.g. tree canopies), and c) coverage of client areas achieved from the base tower height and the potential for improvement with incremental extensions. During the optimisation process performed in this paper, however, only the base tower height of $12 \mathrm{~m}$ is considered, since no actual site inspections are carried out to determine whether sites are capable of hosting taller towers. All viewsheds determined during optimisation in this paper are therefore from a $12 \mathrm{~m}$ observer height above the terrain surface. The visible range that was used in all viewshed analyses is $8 \mathrm{~km}$.

A final factor to consider when determining viewsheds is the curvature of the earth, which becomes more significant and influential in the determining of LOS as the distance from the observer increases. The height at each raster point between the observer and CZ point may be adjusted to simulate the earth's curvature using a method such as Yoeli's correction [65], which is also used in ESRI software. However, at a distance of $8 \mathrm{~km}$ from an observer this correction is minor (approximately $-4 \mathrm{~m}$ ) and thus has not been considered here.

\subsection{Evaluation of CWDSs}

The process of evaluating a CWDS's detection potential is described here. The benchmark Nelspruit system is used as an example and its results are used later for comparison with optimisation results. The evaluation process integrates some elements of the real-world approach that was followed by ForestWatch experts in determining the existing sites.

Two smoke layer heights, such as those illustrated in Fig. 5, were considered above the Nelspruit client area in Fig. 8: $30 \mathrm{~m}$ and $100 \mathrm{~m}^{2}$ A 500-m buffer was

\footnotetext{
${ }^{2}$ The smoke layer heights were raised from $15 \mathrm{~m}$ and $30 \mathrm{~m}$ previously used by Heyns et al. [2] to better reflect practical detection requirements, as advised by ForestWatch decision-makers.
} 
added to the $30-\mathrm{m}$ smoke layer, while a $4-\mathrm{km}$ buffer was added to the $100-\mathrm{m}$ layer. In the actual site-selection process, technicians decided to place six of the towers in Fig. 8 at old watchtower sites and these sites are indicated as "preferred sites." These sites were selected without argument because of the existing infrastructure, road access and historically proven visibility cover. As a result, a thorough site search and comparison of potential sites was only required to determine the twenty remaining towers - according to acceptable terrain conditions, accessibility, and visibility coverage potential [2]. It is accepted that, in a hypothetical green-field exercise with collaboration from ForestWatch experts, the six preferred sites and their associated tower heights would be used. These towers were therefore considered as given, and their coverage with respect to the smoke layers was determined using their actual heights. Since certain parts of the smoke layers are already covered by these towers, the placement of the twenty remaining towers does not require coverage of these areas.

The aim of the optimisation process in this paper is therefore to maximise cover with respect to the remaining uncovered areas, viewed from above in Fig. 10a, b. These areas are the CZs: CZ1 at a $30-\mathrm{m}$ smoke height with a 500-m buffer, and CZ2 at a 100-m smoke height with a 4-km buffer. The twenty existing benchmark

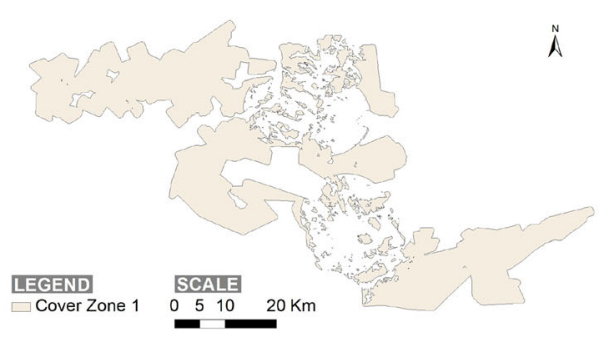

(a)

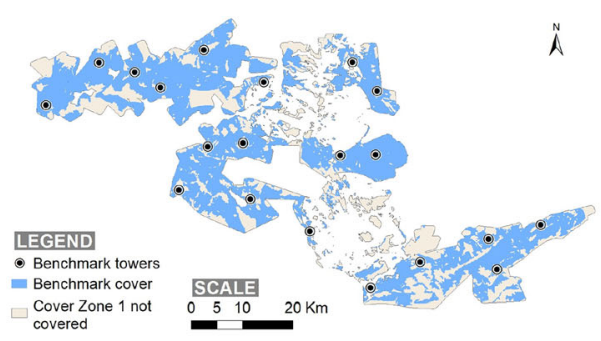

(c)

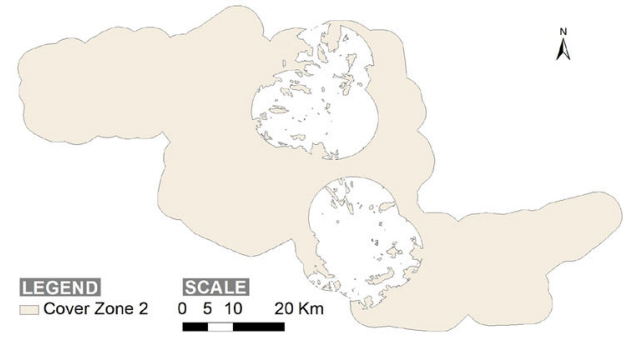

(b)

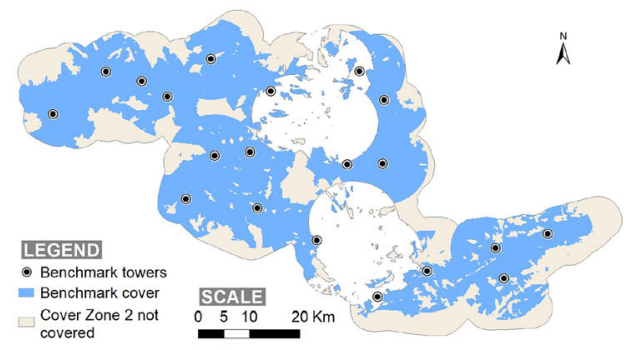

(d)

Figure 10. Cover achieved from six preferred towers is removed from smoke layers at $30 \mathrm{~m}$ and $100 \mathrm{~m}$ (with buffer sizes of $500 \mathrm{~m}$ and $4 \mathrm{~km}$, respectively) above the client area in Fig. 8 and result in (a) CZ1, and (b) CZ2, viewed from above. Cover achieved by the twenty benchmark towers, determined with a detection range of $8 \mathrm{~km}$, is displayed with respect to (c) CZ1 (69.1\%), and (d) CZ2 (66.1\%). 
towers achieve cover of $69.1 \%(\mathrm{CZ1})$ and $66.1 \%$ (CZ2) when evaluated using their actual heights, as shown in Fig. 10c, d.

\subsection{Geomorphon Classification}

The geomorphon classification process (with typical results as previously illustrated in Fig. 3b) was performed for all three regions in Fig. 7 using 30-m SRTM elevation data, and all classifications were processed in the open-source geographic resources analysis support system (GRASS) 7.4.0 software environment. An example of the single line of code used in GRASS to determine geomorphons for a specified digital elevation model (DEM) is the following:

\section{r.geomorphon elevation=input_dem forms=output_geomorphon_dem search=20}

The first expression calls the geomorphon function, the next two expressions set the input and output DEMs, while the final expression sets the lookup distance (previously discussed in Sect. 3.4).

Guided by earlier examples from Jasiewicz and Stepinski [42], a lookup distance of twenty raster points was decided to be sufficient for the purposes of this research, and was also used for the determination of the geomorphons in Fig. 3b. By not specifying any other parameters in the command line, all other parameters are set to the default values of the geomorphon function in GRASS. Descriptions of these parameters and their effects, as well as more detailed information of the geomorphon function are available in the software documentation [47].

The geomorphon DEMs returned by GRASS were exported to raster data for import into ArcGIS software for processing along with all other GIS data previously discussed in Sect. 3.1.

\subsection{Mathematical Problem Formulation}

The mathematical formulation of the problem - which is implemented within an optimisation software environment-is now presented. The aim of maximising demand satisfaction given a fixed number of facilities that are available for placement is known as the maximal covering location problem (MCLP) [66]. In the context of CWDS optimisation, the demand is the visibility cover required by the points in the CZs, while the facilities are the cameras placed on the towers. The CWDS planning problem includes multiple covering objectives, for which a multi-CZ ILP formulation of the MCLP is introduced here. The parameters used are listed below.

\begin{tabular}{ll}
\hline$N_{t}$ & Denotes the number of towers available for placement. \\
$N_{c}$ & Denotes the number of CZs. \\
$s$ & Denotes the index of feasible sites in the PZ. \\
$d_{c}$ & Denotes the index of demand points in $\mathrm{CZ} c$, where $c \in\left\{1, \ldots, N_{c}\right\}$. \\
$N_{d_{c}}$ & Denotes the number of demand points in $\mathrm{CZ} c$. \\
$\mathbb{N}_{d_{c}}$ & Denotes the subset of sites in the PZ from which demand point $d_{c}$ in $\mathrm{CZ} c$ is visible. \\
$x_{s}$ & Is 1 if a tower is placed at site $s$, and 0 otherwise. \\
$y_{d_{c}}$ & Is 1 if demand point $d_{c}$ in $\mathrm{CZ} c$ is covered, and 0 otherwise. \\
\hline
\end{tabular}


The objective can then be written as

$$
\text { maximise } V_{c}=\sum_{d_{c}} y_{d_{c}} \quad \forall c \in\left\{1, \ldots, N_{c}\right\}
$$

where $V_{c}$ is the visibility cover of $\mathrm{CZ} c$, subject to the constraints

$$
\begin{aligned}
& y_{d_{c}} \leq \sum_{s \in \mathbb{N}_{d_{c}}} x_{s} \quad \forall c \in\left\{1, \ldots, N_{c},\right\}, \forall d_{c} \\
& \sum_{s} x_{s}=N_{t} \\
& x_{s} \in\{0,1\} \\
& y_{d_{c}} \in\{0,1\} .
\end{aligned}
$$

The objective in (1) is to maximise cover with respect to each $\mathrm{CZ} c \in\left\{1, \ldots, N_{c}\right\}$. Constraint (2) allows a demand point $d_{c}$ to be covered $\left(y_{d_{c}}=1\right)$ only if one or more cameras are placed at sites in the set $\mathbb{N}_{d_{c}}$. Constraint (3) ensures that exactly $N_{t}$ towers are sited, while constraints (4)-(5) specify binary requirements on the auxiliary variables.

In order to obtain the solutions on the Pareto front that lie between the singleCZ solutions (the end-points on the Pareto front in Fig. 6), the weighted-sum approach is followed. To arrive at the weighted objective function, the $N_{c}$ objectives in (1) are reduced to a single function using a weight, $w_{c}$, for each $\mathrm{CZ}$. The objective is then to

$$
\text { maximise } V=\sum_{c} w_{c} \frac{100}{N_{d_{c}}} \sum_{d_{c}} y_{d_{c}} .
$$

By varying the objective weights in multiple runs, a Pareto front approximation may be traced out. The objective in (6) is subject to the same constraints (2)-(5), enforced with respect to all CZs. The fraction is included in the objective function to reflect the maximisation of the percentage of cover achieved with respect to each $\mathrm{CZ}$, so that the objective function is not biased towards larger CZs with more demand points.

\subsection{Optimisation Process}

As discussed in Sect. 2.5, two stages are followed in the optimisation process followed in this paper. An overview of this process is provided in Fig. 11. The entire process in the figure was followed twice in order to compare the results of optimi- 


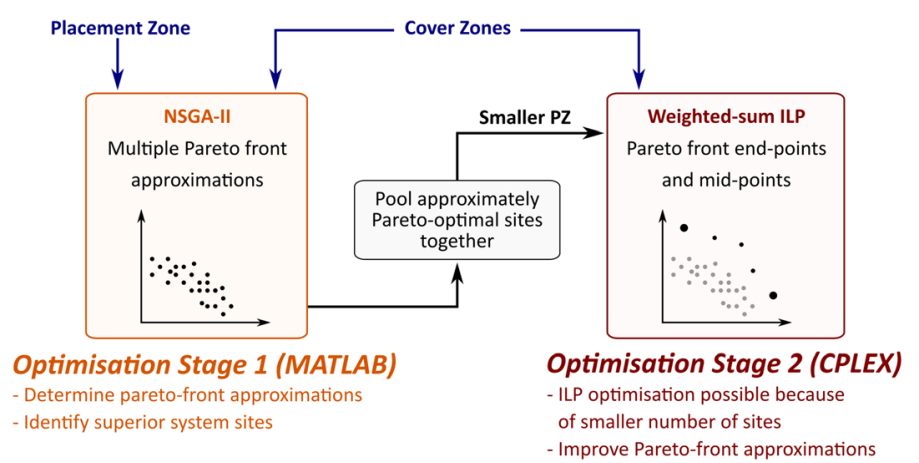

\section{Figure 11. An overview of the optimisation process followed in this paper. In the first stage, the NSGA-II is employed to determine multiple Pareto front approximations. The sites from these approximations are then pooled fogether to form a smaller PZ, which is sufficiently small to be provided as input to an ILP solver, which is employed to determine CWDS layouts of improved quality.}

sation without and with landform exploitation-once using the all-landform PZ in Fig. 4, and a second time using the PZ limited to selected landforms (determined in the results section). In the first stage of the optimisation process, the NSGA-II is employed to determine multiple Pareto front approximations. The sites in the layouts from the multiple Pareto front approximations are then pooled together, resulting in a smaller PZ to provide as input into the ILP stage (the same CZs are again provided as input), solved using the weighted-sum approach and resulting in an improved Pareto front.

The NSGA-II was run using the authors' personal code in MATLAB R2019a. The processing of data to provide as input into CPLEX for ILP optimisation (including the automated generation of its data text file) was also performed in MATLAB. Descriptions of the solution process of the NSGA-II and the parameters that were used for the solutions determined in this paper are available in the "Appendix". CPLEX Studio IDE 12.8.0 was used to solve the weighted-sum objective function in (6). The following weight combinations were used for the two CZs: $(1.00,0.00),(0.75,0.25),(0.5,0.5),(0.25,0.75),(0.00,1.00)$. The first and last weight combinations effectively examine the optimal solution for CZ1 and CZ2, respectively. All computations in this paper were run on a Dell 7820 Precision desktop PC, running Windows 10 Pro with an Intel Xeon Silver 4110 processor and $64 \mathrm{~GB}$ memory.

\subsection{Framework for Analysis and Exploitation of Landforms}

The optimisation process in Fig. 11 forms part of the larger landform analysis and exploitation framework that is followed in this paper. This framework is also proposed as a general process which may be followed in site-selection problems for facilities with similar coverage requirements to those of CWDSs, and using alternative landform classification techniques. A summary of this proposed framework 


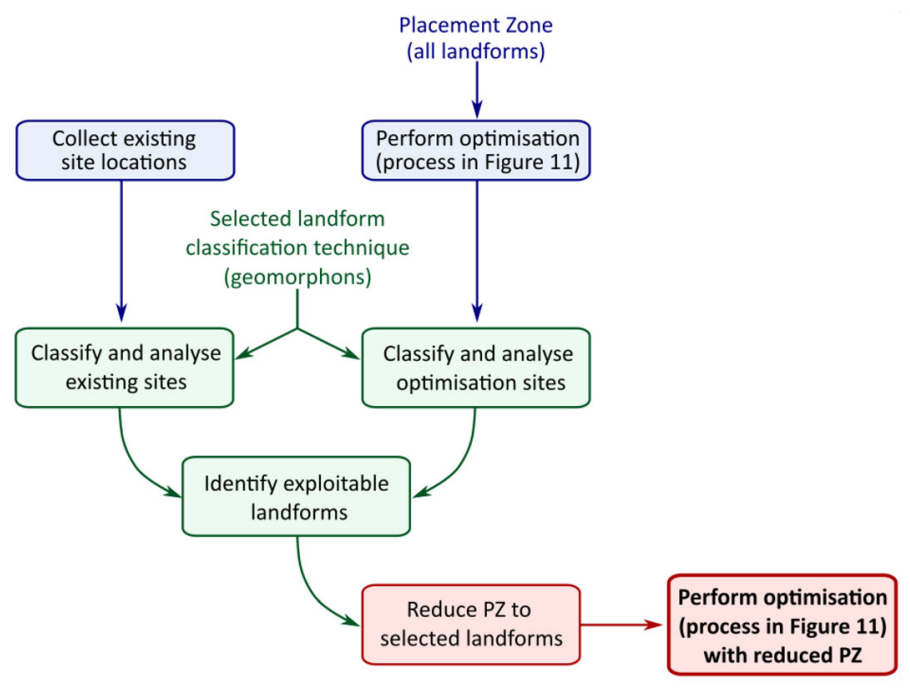

Figure 12. The proposed landform analysis and exploitation optimisation framework, comprising three stages indicated in blue, green, and red. First, existing and optimisation-determined sites are collected (blue), then these sites are analysed using a suitable Iandform classification technique to identify exploitable Iandforms (green). The selected landforms are then exploited to reduce the size of the PZ, leading to improved optimisation results within reduced computation times (red) (Color figure online).

is provided in Fig. 12 and follows three stages - distinguished by colours in blue, green, and red in the figure. The stages are the following:

- Site collection (Blue) The locations of existing facility sites (or historical locations for facilities that are mobile) are collected and, in a separate process, candidate sites are determined by optimisation without landform exploitation (the process in Fig. 11 with the all-landform PZ).

- Landform analysis (Green) A suitable landform classification technique is selected and employed to classify the landforms at the existing/historical site locations and those determined by optimisation. The results of these classifications are examined, with the aim of identifying exploitable overlaps between landforms observed in reality-i.e. real-word decision maker choices and preferences reflected in existing site locations - and computational optimality.

- Landform exploitation (Red) Once exploitable landforms have been identified, the original PZ is reduced to these landforms and the optimisation process in Fig. 11 is repeated with the landform-reduced PZ as input, with the expectation of improved solution quality within reduced computation times.

The framework is now followed in the next section for the study areas introduced in Sect. 3.1. 


\section{Results}

\subsection{Landform Classification Analysis of Existing Sites}

The terrains in Fig. 7 were classified using the geomorphon approach, and the resulting classifications at tower sites are displayed in the bar chart in Fig. 13a. The chart displays the percentage of towers per landform type in blue. As may be seen in the figure, ridges and peaks account for the overwhelming majority of tower landform types. Peaks are the most significant, with $58 \%$ of the tower sites classified as such, while $25 \%$ of the towers are found at ridge sites. To put the tower classifications in the context of their relative surrounding environment, all sites within a 2-km radius of all the towers were also classified and the results are displayed in orange. The radius was chosen as $2 \mathrm{~km}$ because ForestWatch technicians consider alternative sites within such a range when a potential site has been identified. Especially noteworthy is the large ratio of tower sites classified as peaks, compared to the general surrounding terrain also classified as such $-58 \%$ compared to less than $3 \%$. Peaks are clearly sought-after, while ridges are also sought when compared to the surrounding terrain - although to a lesser extent.

A small percentage of towers are observed to be classified other than ridges or peaks (29 towers, or $18 \%$ ). A proximity analysis was performed to further investigate this observation. From each of these towers the distance to the nearest ridge or peak site was calculated. It was determined that 17 of the 29 towers are no further than $45 \mathrm{~m}$ from a peak or a ridge, while 27 are no further than $95 \mathrm{~m}$. Complete results are displayed as a histogram in Fig. 13b. From discussions with ForestWatch technicians, the explanation for this is that practical considerations such as rocky and jagged ground surface conditions and challenging journeys from roads result in desirable peak and ridge sites sometimes being sacrificed for less challenging sites nearby which still offer good visibility. The information encapsulated in Fig. 13 therefore reveal that the sites selected by ForestWatch experts favour peaks and ridges (according to the geomorphon classification used in this paper).

The primary conclusion from this analysis is that all 165 towers are either placed at or very near to ridges and peaks, and as a result, limiting the site search to geomorphon-classified peak and ridge landforms will provide decision makers with sites that reflect their preferences and are either (a) practical and ultimately implementable, or (b) sufficiently close to suitable alternatives.

\subsection{Optimisation and Analysis with all Landforms}

Fifteen NSGA-II Pareto front approximations were generated using as input the PZ in Fig. 4 which has not been limited to specific landforms. A total of 2097 solutions were returned by the approximations and are indicated by the orange crosses in Fig. 14. Further investigation revealed that these solutions comprise different combinations of 564 unique sites.

The 564 unique sites were then used as a new set of candidate sites for ILP optimisation. The weighted optimal solutions are displayed as red crosses in Fig. 14. Further analyses revealed that the five ILP solutions are unique combina- 


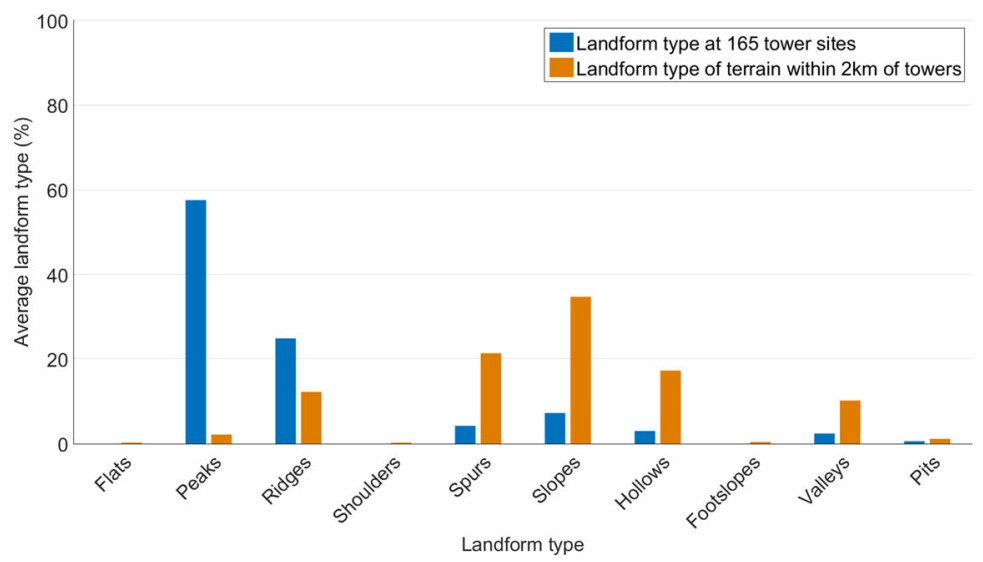

(a)

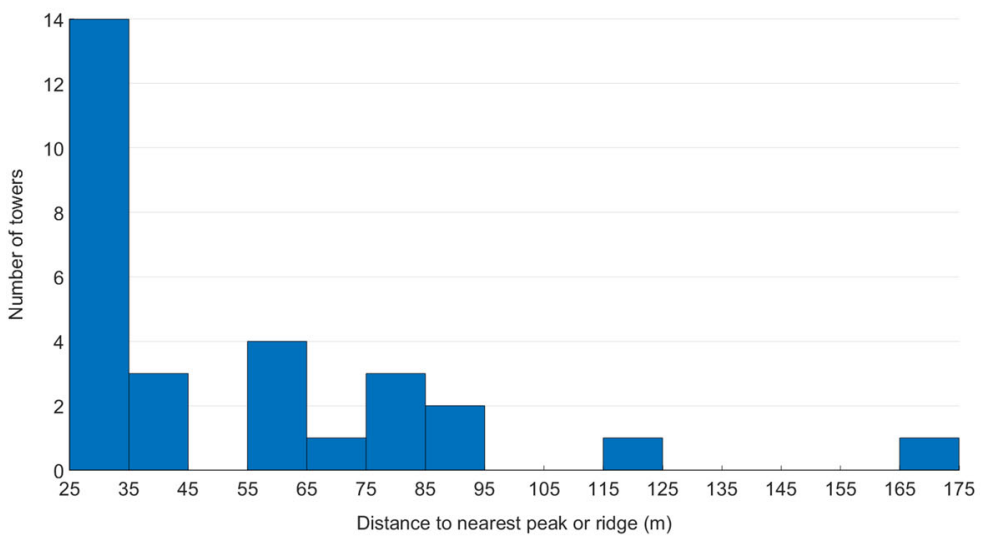

(b)

Figure 13. (a) Landform type percentages of 165 towers and Iandform type percentages of all terrain within a $2 \mathrm{~km}$ radius from all the fowers. (b) Histogram showing the distances of towers that are not classified as ridges or peaks from the nearest points that are classified as ridges or peaks.

tions of 47 sites. The total heuristic computation time was $174 \mathrm{~h}$ and $40 \mathrm{~min}$, while the ILP computation time of the five weighted runs totalled $3 \mathrm{~h}$ and $7 \mathrm{~min}$.

The classifications of the constituent sites of the all-landform optimisation results are displayed in Fig. 15 as a bar chart of the average percentage per landform type. Peaks and ridges dominate the 564 sites obtained by the heuristic runs, as well as the 47 sites from weighted optimisation. Specifically, $87 \%$ of the sites identified by the heuristics were either peaks or ridges. Even more dramatically, of the 47 sites contained in the five weighted optimal solutions, 94\% are classified as peaks or ridges. Only three of these 47 sites are not peaks and ridges, and these 


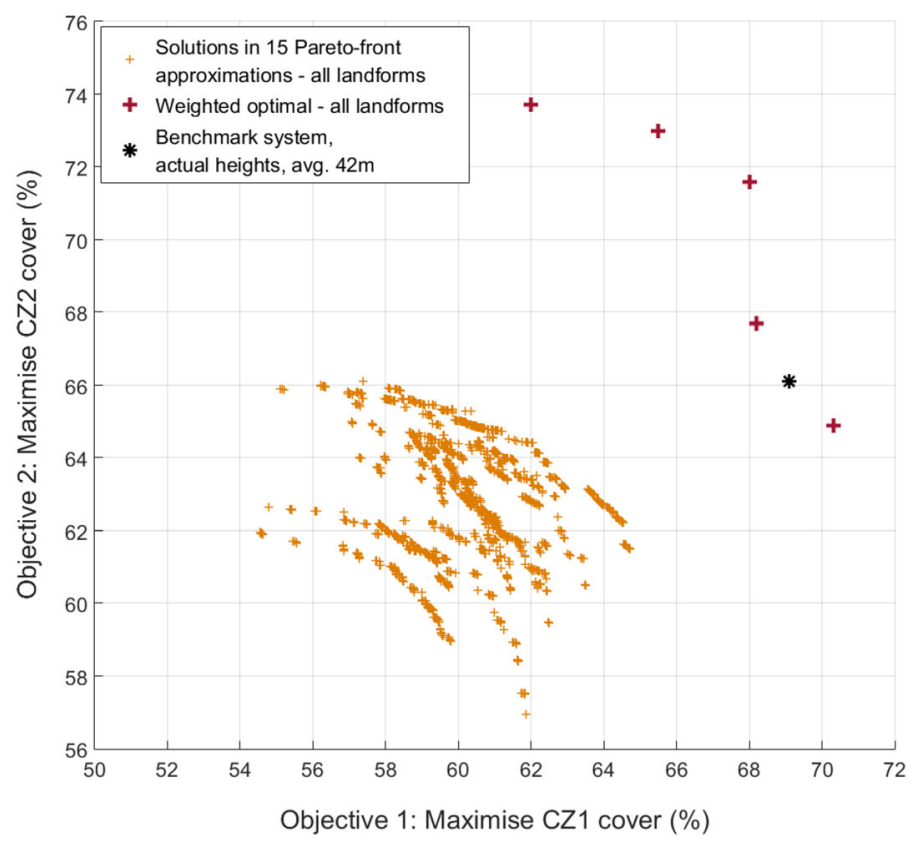

Figure 14. Results in objective function space of multiple heuristic runs (the orange crosses), and subsequent weighted optimal runs (the red crosses), determined with respect to the all-landform PZ in Fig. 4 (Color figure online).

three sites are all classified as spurs. Further investigation revealed that all three were directly adjacent to a ridge site.

Figure 16 shows the distance to the nearest peak or ridge site as measured from sites that are not classified as peaks or ridges, for the sites in the heuristic and weighted solutions respectively. For the heuristic runs, 63 of the 72 sites that were not peaks or ridges were within $50 \mathrm{~m}$ of a peak or ridge, as were the three ILP-determined spur sites mentioned above. Based on the information provided in Figs. 15 and 16 it may be concluded that, even when the candidate sites are not limited to specific landforms, the final sites determined by the optimisation process are destined to arrive at geomorphon-classified peaks and ridges, or close.

\subsection{Optimisation and Analysis After Landform Exploitation}

In the above sections, peaks and ridges were identified as exploitable landforms based on the existing tower-site landform analysis and the results of all-landform optimisation. The PZ in Fig. 4 is now limited to geomorphon-classified peaks and ridges only and the result is displayed in Fig. 17. Compared to the original PZ which comprises 741,813 candidate sites, the new PZ comprises only 146,874 sites - a significant reduction of $80 \%$ in the number of candidate sites. 


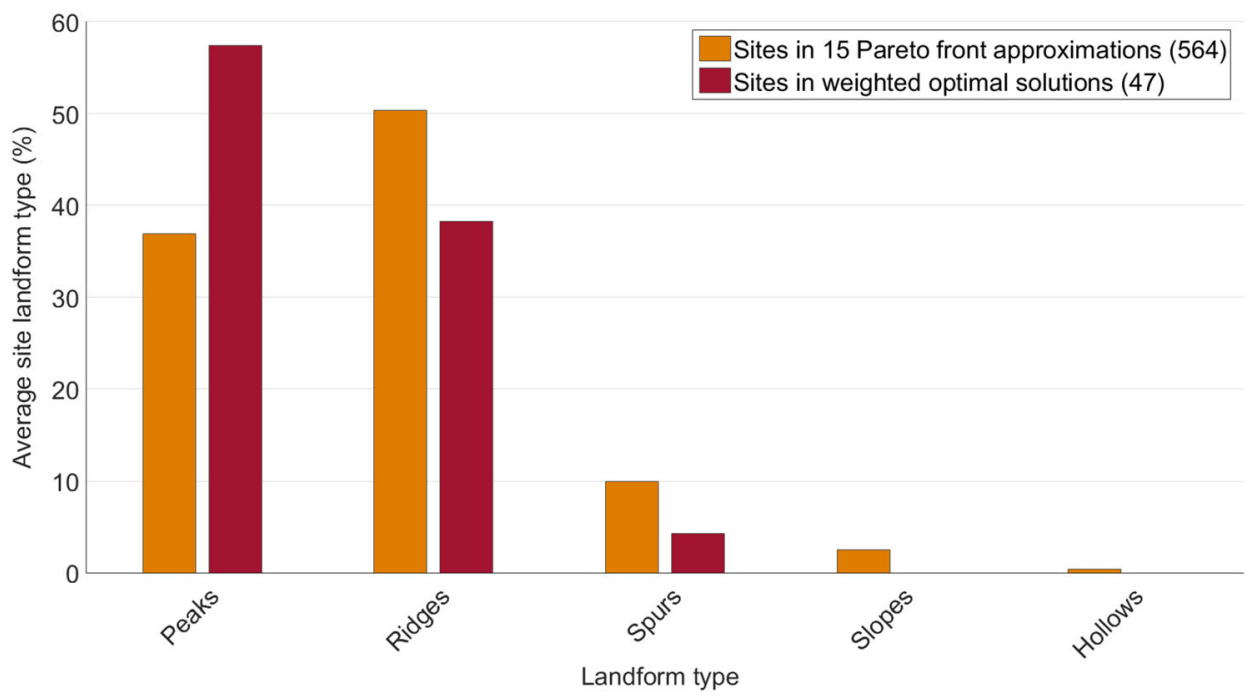

Figure 15. Average percentage of sites per landform type per optimisation stage for the solution process followed with all landform types.

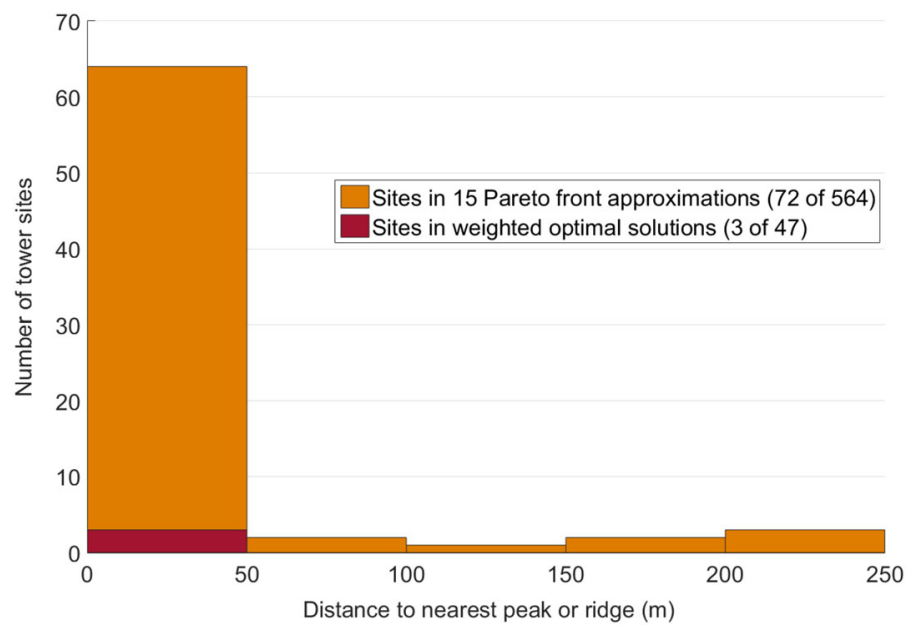

Figure 16. Distances of sites that are not classified as ridges or peaks (the spurs, slopes and hollows in Fig. 15) from the nearest points that are classified as ridges or peaks, per optimisation stage.

The optimisation process in Fig. 11 was repeated, now using the landform-limited PZ as input - using the same heuristic parameters and ILP weight sets as before. Fifteen Pareto front approximations obtained by the heuristic produced 2138 solutions - indicated by the grey circles in Fig. 18 - which are combinations 


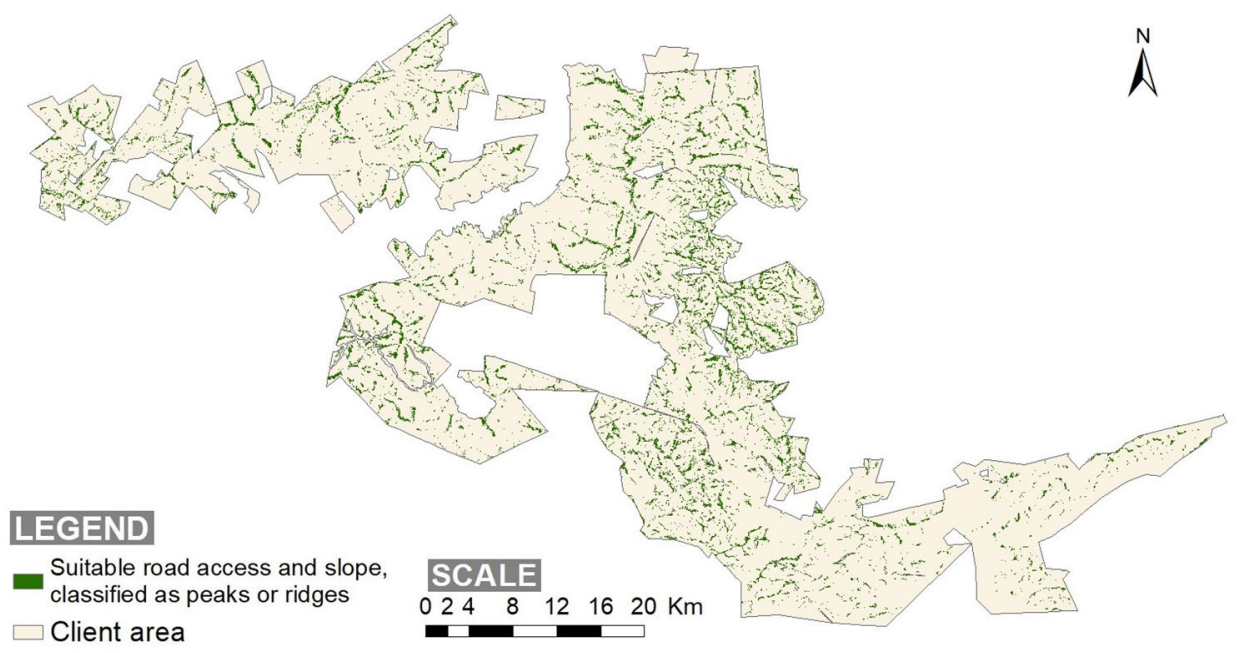

\section{Figure 17. Update of the PZ in Fig. 4, limited to peaks and ridges.}

of 476 unique sites. The solutions previously obtained by the all-landform PZ optimisation process, in Fig. 14, are also displayed in Fig. 18 for comparison purposes. The 476 unique sites from the landform-limited heuristic runs were then used as input for weighted ILP runs, and the resulting solutions are displayed as black circles in Fig. 18. The five solutions are unique combinations of 42 sites.

The total heuristic computation time using the new PZ was $149 \mathrm{~h}$ and $34 \mathrm{~min}$,while the ILP computation time of the five weighted runs totalled $1 \mathrm{~h}$ and $59 \mathrm{~min}$.

\section{Discussion}

A comparison between the results of optimisation with and without the exploitation of landforms in Fig. 18 provides evidence that landform classification is beneficial to the planning of CWDS layouts - without compromising solution quality and achieved within reduced computation times. Comparing the results of the heuristic runs, conspicuous improvement in objective function values is observed in Fig. 18 when using peaks and ridges only. This was achieved in $149 \mathrm{~h}$ and $34 \mathrm{~min}$, which is $25 \mathrm{~h}(14.3 \%)$ faster than the all-landform runs. Comparing the weighted optimal solutions, no deterioration in solution quality is apparent by limiting the candidate sites to peaks and ridges, and in some cases, the solution quality may be improved. However, a significant reduction in computation time of $1 \mathrm{~h} 8 \mathrm{~min}(36.4 \%)$ was observed.

Of particular note is that one weighted solution from the peaks/ridges-only PZ dominates the benchmark system with respect to both objectives - significant because the optimisation approach only locates $12-\mathrm{m}$ towers, while the benchmark system is evaluated with actual tower heights that average $42 \mathrm{~m}$. The installation cost of a $12-\mathrm{m}$ tower is more than three times less than that of a $42-\mathrm{m}$ tower, ${ }^{3}$

\footnotetext{
${ }^{3}$ Determined from tower installation costs provided by ForestWatch technicians.
} 


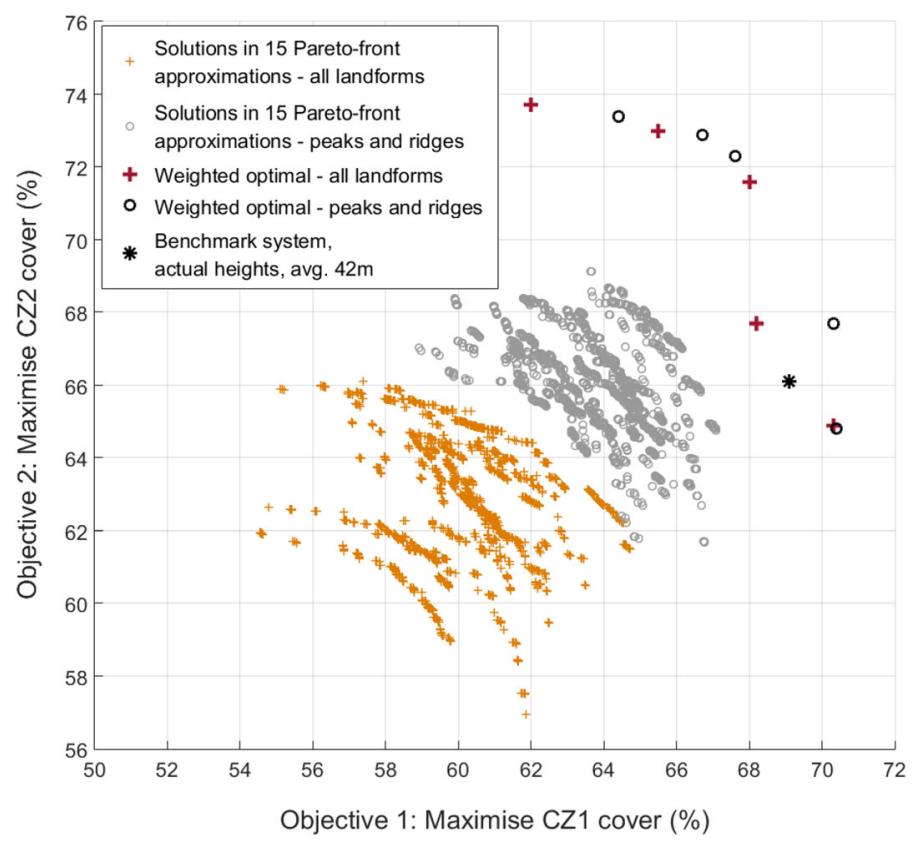

Figure 18. Results in objective function space of multiple heuristic runs (the orange crosses and grey circles), and subsequent weighted optimal runs (the red crosses and black circles). Solutions that are orange and red crosses were determined with respect to the original PZ in Fig. 4, while solutions that are grey and black circles were determined with respect to the same PZ reduced to peaks and ridges only (Fig. 17 ) (Color figure online).

indicating substantial potential cost savings if the optimisation approach is followed in future CWDS planning. However, the optimisation approach does not take vegetation growth around the sites into account (the terrain model only includes surface elevation), and increases in tower heights above $12 \mathrm{~m}$ may still be required to rise above vegetation, depending on site inspections. Nevertheless, the same terrain model is used to determine the visibility of the benchmark towers at an average of $42 \mathrm{~m}$ and the optimisation-determined solutions at $12 \mathrm{~m}$.

The effect of vegetation interference (such as tree canopies) surrounding a tower site is a factor that plays a deciding role in the height of a tower, but is problematic to implement within the optimisation framework. This is because the vegetation around the towers regularly changes in this environment (forestry compartments being harvested or vegetation cleared for fire management, etc.) and because vegetation interference also depends on the terrain surrounding a tower. This is because trees with a high canopy will interfere more with a tower's visibility if the area surrounding the tower is flat, than if trees with the same canopy height are found on slopes running downward from the tower-in which case only the immediately surrounding trees have a noteworthy impact on the 
tower's visibility. In practice, trees surrounding a future tower site are sometimes cleared out to make space for the required structures, which also improves visibility. Including vegetation data is therefore not advised, because of its ever-changing nature. Instead, using the shortest tower height in the optimisation process is the best approach, because the returned results should be considered worst-case scenario. Increasing tower heights at proposed sites is inevitable, and these increases only improve the results calculated for 12-m towers once obstructions are cleared.

The resolution of terrain data used plays a role in the optimisation process. Here, 30-m SRTM data were used. Higher data resolutions are available, and offer improved accuracy in terms of the terrain representation and associated visibility computations. Higher resolution data can help to detect finer details in the terrain's topography and smaller visibility obstructions that can be detected by higher-resolution satellite measurements and which may be overlooked at lower resolutions (such as a boulder or local elevated area nearby a candidate site). It has been shown how a DEM's resolution plays a significant role in a site's visibility computations, particularly in its nearby vicinity [64]. The detection of such obstructions can play a significant role in the candidate site's visibility computations, and the overall visibility determined for a CWDS layout. Nevertheless, even if such nearby obstructions may be determined at higher resolutions, their impact may not be problematic in reality because the tower can simply be raised to overlook such an obstruction. These obstructions may therefore be more of a concern in terms of the terrain's suitability for tower installation and access and the use of higher resolution should therefore be preferred for these reasons. However, higher resolution data results in increased computational complexity because of the larger number of candidate sites. This provides further motivation to implement the research presented in this paper so that the number of candidate sites can be effectively reduced to a manageable number.

Different landform classification techniques exist which may be employed in the landform exploitation framework. The geomorphon approach was selected here because of its simplicity and availability in open-source software. The implementation of other techniques is certainly possible, and depends on user preference. As long as the stages in the framework of Fig. 12 are followed, it should be possible to emphasise desireable sites regardless of which classification technique is employed - with the end result being that the landform-limited PZ returns solutions that are superior, yet practical, compared to using a PZ that contains all landforms.

The parameters of each technique are similarly of interest, as they may return markedly different results. Consider, for example, the lookup distance specified for the geomorphon approach (discussed in Sect. 3.4). A lookup distance of twenty raster cells was used here. In post-analysis, the terrain was again classified using a lookup distance of ten raster cells. When analysing the 42 sites in the weighted sum solutions that were returned for the peaks/ridges-only PZ, 41 of these were also classified as peaks or ridges using the classes returned with the smaller lookup distance. The one site that was classified otherwise was a spur, with 4 of its neighbouring sites classified as ridges. Using a lookup distance of ten cells would there- 
fore return similar results since the superior sites remain included and the one site may be substituted by an immediate neighbour by the optimisation process. However, what is noteworthy is that the PZ limited to peaks and ridges using the smaller lookup distance is comprised of 108,314 candidate sites, compared to the 146,874 in the PZ using a lookup distance of twenty. Therefore, similar results in solution quality may be achieved, but with reduced complexity and within reduced computation times due to a decrease of $38,560(26 \%)$ in the number of candidate sites. Similar improvements may be possible upon inspection of the effects of variations in other parameters, or by implementing other classification techniques.

The heuristic stage of the optimisation process followed in this paper is essential for reducing the number of candidate sites so that improved results are possible with the second optimisation stage. The NSGA-II is one of many algorithms that may be used for this purpose. While the NSGA-II has been used in various similar problems and has achieved good results [2, 18, 20, 56, 59], alternatives may be investigated to reveal if better solutions can be discovered (also considering computation time). Examples include simulated annealing [67], the strength-pareto evolutionary algorithm 2 [68], and the hybrid geospatial algorithm [61].

\section{Conclusion}

The implementation of landform classes to improve CWDS tower-site selection optimisation was investigated. A preliminary analysis categorised 165 existing towers from three ForestWatch CWDSs according to geomorphon landform types - the first time such a classification has been performed for actual sites of facilities with visibility-based objectives. It was found that 136 (or $82 \%$ ) of these towers were sited at peak or ridge sites, while those that are sited otherwise are never far away from peaks or ridges. Optimisation methods were then employed to determine candidate CWDS layouts for a forestry area in the Nelspruit region of South Africa, currently monitored by an existing ForestWatch CWDS - taking as input candidate sites that were not limited to specific landforms. The results complemented those of the practical tower classification exercise; peaks and ridges made up $87 \%$ of the sites identified by the heuristic stage, while $94 \%$ of the sites in the weighted optimal solutions were classified as peaks or ridges. Based on these results, the PZ was reduced to geomorphon-classified peaks and ridges only. While the original all-landform PZ comprised 741,813 candidate sites, it was possible to achieve an $80 \%$ reduction in the number of candidate sites to 146,874 when considering peaks and ridges only.

The optimisation results provided clear evidence that landform classification is beneficial to the planning of CWDS layouts without compromising solution quality (in fact, indicating potential improvement in solution quality), while significant reduction in computation times was achieved. Furthermore, the analyses performed in this paper revealed the practical functionality of implementing landform exploitation. The selected landforms, of course, depend on the landform classification technique employed - and could also depend on the type of facility investigated - and other classification techniques may suggest the selection of different landforms. A notable benefit of the landform exploitation framework presented 
here is that it may be employed with various terrain types (rough, flat, etc.), as long as a suitable landform classification approach and parameter settings are employed.

The work presented here may be easily replicated for use with other applications that require the placement of facilities with line-of-sight-based maximisation objectives. Examples from the literature in which site-selection approaches were followed that may well have benefited from the work presented here include military equipment such as signal jammers [16] and radars and weapons [17], cellular transmitters [11-13], and weather radar [14, 15]. Caution should, however, be taken to avoid the haphazard selection of landform types to consider for placement, while the landform classification technique to employ also requires investigation. The hope is that the framework proposed and followed in this paper (Fig. 12) provides a guideline for such replication.

\section{Acknowledgements}

Opinions expressed and conclusions arrived at are those of the authors and are not necessarily to be attributed to the CoE-MaSS.

\section{Funding}

Open access funding provided by Hanken School of Economics.

\section{Declarations}

Conflict of interest The authors declare that they have no conflict of interest.

\section{Open Access}

This article is licensed under a Creative Commons Attribution 4.0 International License, which permits use, sharing, adaptation, distribution and reproduction in any medium or format, as long as you give appropriate credit to the original author(s) and the source, provide a link to the Creative Commons licence, and indicate if changes were made. The images or other third party material in this article are included in the article's Creative Commons licence, unless indicated otherwise in a credit line to the material. If material is not included in the article's Creative Commons licence and your intended use is not permitted by statutory regulation or exceeds the permitted use, you will need to obtain permission directly from the copyright holder. To view a copy of this licence, visit http://creat ivecommons.org/licenses/by/4.0/. 


\section{Appendix}

The NSGA-II is a genetic algorithm, and a candidate CWDS layout is represented as a chromosome string of feasible tower site numbers [58, 69]. The site numbers are pre-determined by an indexing scheme-typically derived with respect to row and column indices - for all the sites within the PZ's raster representation [58]. A chromosome 23-120-8779-13065, for example, represents a candidate CWDS with four towers located at sites 23, 120, 8779 and 13065. Evolution-inspired population progression processes and chromosome modification operators are performed on a randomly generated population of such candidate CWDS chromosomes until some termination criterion is met [69]. A typical termination criterion is when the successive populations reach a point where they fail to significantly improve on the solution quality of previous generations [61]. Solutions which perform well with respect to the objective functions are normally selected for modification as "parents"-meaning that the offspring solutions typically exhibit some of the strong properties of their parents.

Two mechanisms are utilised by the NSGA-II to explore new solutions. Crossovers are performed between sub-strings of parent chromosomes and result in new offspring solutions that consist of new site combinations that are inherited from the parent solutions [58, 69]. Parents are selected for crossover from a smaller pool of randomly selected solutions from the population (called a tournament pool), and superior solutions from the smaller pool are selected for crossover-resulting that the offspring solutions typically exhibit some of the strong properties of their parents. Not all parent solutions chosen by tournament selection, however, undergo crossover. Instead, crossover is subject to a crossover probability [69]. Mutation follows crossover and promotes site diversity by stochastically introducing new, unexplored site locations into the chromosomes, instead of simply exchanging already explored sites by means of crossover [58, 69]. Mutation occurs for each offspring solution from the crossover process with a mutation probability.

For readers interested in the specific parameters, the following values were used. The population size was chosen as 1400 , the tournament size was 4 , the crossover probability was 0.9 and the mutation probability was 0.2 (which is unusually high). Because crossover does not alter the sites in a solution combination (only the combination), it lacks the inherent mutation that is present in traditional binary representations [70]. The unusually high mutation probability after crossover therefore compensates for this.

\section{References}

1. Martell DL (2015) A review of recent forest and wildland fire management decision support systems research. Curr For Rep 1:128-137

2. Heyns AM, du Plessis W, Kosch M, Hough G (2019) Optimisation of tower site locations for camera-based wildfire detection systems. Int J Wildland Fire 28(9):651-665 
3. Franklin WR, Clark R (1994) Higher isn't necessarily better: visibility algorithms and experiments. In: Advances in GIS research: sixth international symposium on spatial data handling. Taylor \& Francis, pp 751-770

4. Nagy G (1994) Terrain visibility. Comput Graphics 18(6):763-773

5. Lee J (1994) Digital analysis of viewshed inclusion and topgraphic features on digital elevation models. Photogramm Eng Remote Sens 60(4):451-456

6. Rana S (2003) Fast approximation of visibility dominance using topographic features as targets and the associated uncertainty. Photogramm Eng Remote Sens 69(8):881-888

7. Kim YH, Rana S, Wise S (2004) Exploring multiple viewshed analysis using terrain features and optimisation techniques. Comput Geosci 30(9-10):1019-1032

8. Bao S, Xiao N, Lai Z, Zhang H, Kim C (2015) Optimizing watchtower locations for forest fire monitoring using location models. Fire Saf J 71:100-109

9. Zhang F, Zhao P, Thiyagalingam J, Kirubarajan T (2019) Terrain-influenced incremental watchtower expansion for wildfire detection. Sci Total Environ 654:164-176

10. Eugenio FC, Rosa dos Santos A, Fiedler NC, Ribeiro GA, da Silva AG, Juvanhol RS, Schettino VR, Marcatti GE, Domingues GF, Alves dos Santos GMAD, Pezzopane JEM, Pedra BD, Banhos A, Martins LD (2016) GIS applied to location of fires detection towers in domain area of tropical forest. Sci Total Environ 562:542-549

11. Krzanowski R, Raper J (1999) Hybrid genetic algorithm for transmitter location in wireless networks. Comput Environ Urban Syst 23(5):359-382

12. Akella MR, Delmelle E, Batta R, Rogerson P, Blatt A (2010) Adaptive cell tower location using geostatistics. Geogr Anal 42(3):227-244

13. Mathar R, Niessen $T$ (2000) Optimum positioning of base stations for cellular radio networks. Wirel Netw 6(6):421-428

14. Minciardi R, Sacile R, Siccardi F (2003) Optimal planning of a weather radar network. J Atmos Ocean Technol 20(9):1251-1263

15. Domenikiotis C, Dalezios NR, Faraslis I (2010) GIS-based weather radar siting procedure in mountainous terrain. Phys Chem Earth Parts A/B/C 35:35-42

16. Gencer C, Aydogan EK, Celik C (2008) A decision support system for locating VHF/ UHF radio jammer systems on the terrain. Inf Syst Front 10(1):111-124

17. Tanergüclü T, Mara s H, Gencer C, Aygüneş H, (2010) A decision support system for locating weapon and radar positions in stationary point air defence. Inf Syst Front $14: 423-444$

18. Heyns AM, Van Vuuren JH (2018) Multi-type, multi-zone facility location. Geogr Anal 32(11):1434-1444

19. Franklin WR (2002) Siting observers on terrain. In: Richardson DE, van Oosterom P (eds) Advances in spatial data handling Springer, Berlin, pp 109-120

20. Kwong WY, Zhang PY, Romero D, Moran J, Morgenroth M, Amon C (2014) Multiobjective wind farm layout optimization considering energy generation and noise propagation with NSGA-II. J Mech Des 136(9):1-10

21. Heyns AM, Van Vuuren JH (2015) An evaluation of the effectiveness of observation camera placement within the MeerKAT radio telescope project. S Afr J Ind Eng 26:110

22. Farr TG, Rosen PA, Caro E, Crippen R, Duren R, Hensley S, Kobrick M, Paller M, Rodriguez E, Roth L, Seal D, Shaffer S, Shimada J, Umland J, Werner M, Oskin M, Burbank D, Alsdorf D (2007) The shuttle radar topography mission. Rev Geophys . https://doi.org/10.1029/2005RG000183

23. Greysukh VL (1967) The possibility of studying landforms by means of digital computers. Soviet Geogr 8:137-149 
24. Johnston EG, Rosenfeld A (1975) Digital detection of pits, peaks, ridges, and ravines. IEEE Trans Syst Man Cybern SMC-5:472-480

25. Peucker TK, Douglas DH (1975) Detection of surface-specific points by local parallel processing of discrete terrain elevation data. Comput Graphics Image Process 4:375387

26. Schillaci C, Braun A, Kropáček J (2015) Terrain analysis and landforms recognition. In: Geomorphological techniques. British Society for Geomorphology, online edition ed., pp 1-18

27. Lee J, Snyder P, Fisher P (1992) Modeling the effect of data errors on feature extraction from digital elevation models. Photogramm Eng Remote Sens 58(10):1461-1467

28. Schmidt J, Hewitt A (2004) Fuzzy land element classification from DTMs based on geometry and terrain position. Geoderma 121:243-256

29. Schmidt J, Andrew R (2005) Multi-scale landform characterization. Area 37(3):341-350

30. O'Callaghan JF, Mark DM (1984) The extraction of drainage networks from digital elevation data. Comput Vis Graphics Image Process 28:323-344

31. Skidmore AK (1989) A comparison of techniques for calculating gradient and aspect from a gridded digital elevation mode. Int J Geogr Inf Syst 3:323-334

32. Smith MJ, Rose J, Gousie MB (2009) The cookie cutter: a method for obtaining a quantitative 3D description of glacial bedforms. Geomorphology 108:209-218

33. Jenson SK, Domingue JO (1988) Extracting topographic structure from digital elevation data for geographic information system analysis. Photogramm Eng Remote Sens 54:1593-1600

34. Band LE (1986) Topographic partition of watersheds with digital elevation models. Water Resour Res 22:15-24

35. Grohmann CH, Smith MJ, Riccomini C (2010) Multiscale analysis of topographic surface roughness in the Midland Valley, Scotland. Geosci Remote Sens 49:1-14

36. Wieczorek GF, Snyder JB (2009) Monitoring slope movements. In: Young R, Norby L (eds) Geological monitoring Geological Society of America, Boulder, pp 245-271

37. Zakerinejad R, Märker M (2014) Prediction of gully erosion susceptibilities using detailed terrain analysis and maximum entropy modeling: a case study in the Mazayejan Plain, Southwest Iran. Geogr Fis Din Quat 37:67-76

38. Romero BE, Clarke KC (2018) Exploring uncertainties in terrain feature extraction across multi-scale, multi-feature, and multi-method approaches for variable terrain. Cartogr Geogr Inf Sci 45:381-399

39. Stepinski TF, Jasiewicz J (2011) Omorphons - a new approach to classification of landforms. In: Proceedings of geomorphometry 2011. Redlands, CA, USA, pp 109-112

40. Dikau R (1989) The application of of a digital relief model to landform analysis in geomorphology. In: Three dimensional application in geographic information systems. Taylor \& Francis, London, pp 51-77

41. Wood J (1996) The geomorphological characterisation of digital elevation models. Ph.D. Thesis, University of Leicester, Leicester, UK

42. Jasiewicz J, Stepinski TF (2013) Geomorphons - a pattern recognition approach to classification and mapping of landforms. Geomorphology 182:147-156

43. Di Stefano M, Mayer L (2018) An automatic procedure for the quantitative characterization of submarine bedforms. Geosciences 8:28

44. Harmon BA, Petrasova A, Petras V, Mitasova H, Meentemeyer R (2018) Tangible topographic modeling for landscape architects. Int J Archit Comput 16:4-21

45. Djurdjevac Conrad N, Helfmann L, Zonker J, Winkelmann S, Schütte C (2018) Human mobility and innovation spreading in ancient times: a stochastic agent-based simulation approach. EPJ Data Sci 7(1):1-12 
46. Luo W, Liu CC (2018) Innovative landslide susceptibility mapping supported by geomorphon and geographical detector methods. Landslides 15:465-474

47. GRASS GIS manual (2020) "r.geomorphon"

48. Hough G (2007) Vision systems for wide area surveillance: ForestWatch-a long-range outdoor wildfire detection system. In: TASSIE FIRE conference proceedings. Hobart, Tasmania

49. Tabik S, Zapata EL, Romero LF (2013) Simultaneous computation of total viewshed on large high resolution grids. Int J Geogr Inf Sci 27(4):804-814

50. Misthos LM, Nakos B, Krassanakis V, Menegaki M (2018) The effect of topography and elevation on viewsheds in mountain landscapes using geovisualization. Int J Cartogr 5:44-66

51. Zitzler E, Deb K, Thiele L (2000) Comparison of multiobjective evolutionary algorithms: empirical results. Evol Comput 8(2):173-195

52. Machairas V, Tsangrassoulis A, Axarli K (2014) Algorithms for optimization of building design: a review. Renew Sustain Energy Rev 31:101-112

53. Xia J, Curtin KM, Huang J, Wu D, Xiu W, Huang Z (2019) A carpool matching model with both social and route networks. Comput Environ Urban Syst 75:90-102

54. Yao J, Zhang X, Murray AT (2018) Spatial optimization for land-use allocation: accounting for sustainability concerns. Int Region Sci Rev 41:579-600

55. Cohon JL (1978) Multiobjective programming and planning. Academic Press, New York

56. Kim K, Murray AT, Xiao N (2008) A multiobjective evolutionary algorithm for surveillance sensor placement. Environ Plan 35(5):935-948

57. Jia H, Ordóñez F, Dessouky MM (2007) Solution approaches for facility location of medical supplies for large-scale emergencies. Comput Ind Eng 52(2):257-276

58. Heyns AM, van Vuuren JH (2016) A multi-resolution approach towards point-based multi-objective geospatial facility location. Comput Environ Urban Syst 57:80-92

59. Raisanen L, Whitaker RM (2005) Comparison and evaluation of multiple objective genetic algorithms for the antenna placement problem. Mob Netw Appl 10(1-2):79-88

60. Mavrotas G (2009) Effective implementation of the epsilon-constraint method in multiobjective mathematical programming problems. Appl Math Comput 213(2):455-465

61. Heyns AM (2016) A multi-objective approach towards geospatial facility location. Author Ph.D. Thesis, Stellenbsoch University, Stellenbosch

62. Floriani L, Magillo P (2003) Algorithms for visibility computation on terrains: a survey. Environ Plan 30(5):709-728

63. Bresenham JE (1965) Algorithm for computer control of digital plotter. IBM Syst J 4(1):25-30

64. Heyns AM, Van Vuuren JH (2013) Terrain visibility-dependent facility location through fast dynamic step-distance viewshed estimation within a raster environment. In: Proceedings of the 2013 annual conference of the operations research society of South Africa, pp 112-121

65. Yoeli P (2006) The making of intervisibility maps with computer and plotter. Cartogr Int J Geogr Inf Geovis 22:88-103

66. Church R, ReVelle C (1974) The maximal covering location problem. Pap Region Sci Assoc 32:101-118

67. Smith K, Everson R, Fieldsend J (2004) Dominance measures for multi-objective simulated annealing. In: Proceedings of the 2004 congress on evolutionary computation (IEEE Cat. No.04TH8753). IEEE, Portland, OR, USA, pp 23-30

68. Zitzler E, Laumanns M, Thiele L (2001) SPEA2: improving the strength pareto evolutionary algorithm. Technical Reports. TIK-Report 103, ETH Zurich 
69. Deb K, Pratap A, Agarwal S, Meyarivan T (2002) A fast and elitist multiobjective genetic algorithm: NSGA-II. IEEE Trans Evol Comput 6(2):182-197

70. De Jong KA, Spears WM (1992) A formal analysis of the role of multi-point crossover in genetic algorithms. Ann Math Artif Intell 5:1-26

Publisher's Note Springer Nature remains neutral with regard to jurisdictional claims in published maps and institutional affiliations. 